\title{
Mass spectrometry imaging of rat brain lipid profile changes over time
}

\section{following traumatic brain injury}

Aurelie Roux ${ }^{1}$; Ludovic Muller ${ }^{1}$; Shelley N Jackson ${ }^{1}$; Jeremy Post ${ }^{1}$; Katherine Baldwin ${ }^{1}$; Barry Hoffer ${ }^{5}$; Carey D Balaban ${ }^{4}$; Damon Barbacci ${ }^{3}$ J. Albert Schultz ${ }^{3}$; Shawn Gouty ${ }^{2}$; Brian M Cox ${ }^{2}$; Amina S.

$$
\text { Woods }^{1 *}
$$


Traumatic brain injury (TBI) is damage to the brain that temporarily or permanently impairs brain structure and functions. It is a serious public health problem and results in many permanent disabilities. The CDC reported that over 1.7 million people sustain TBI every year in the United States, which accounts for about a third (30.5\%) of all injury-related deaths (Faul et al., 2010). Even non-lethal TBI causes a wide range of short and long term functional changes including sensory and cognitive impairments involving memory, movement, vision, hearing, as well as emotional dysfunctions, personality changes and depression (Rao and Lyketsos, 2000;Kushner, 1998). Moreover TBI increases risks of epilepsy (Agrawal et al., 2006) and other brain disorders with symptoms resembling Alzheimer's and Parkinson's diseases (National Institute of Neurological Disorders and Stroke., 2002). The pathophysiology of TBI involves two stages: (1) the primary injury occurring at impact resulting in direct tissue damage and impairment of cerebral blood flow and metabolism, (Hardman and Manoukian, 2002), and (2) the secondary injury resulting from biochemical and pathological processes initiated by the mechanical damage (Hardman and Manoukian, 2002). Secondary injuries such as cerebral ischemia, intracranial hypertension, inflammation and edema are the leading causes of hospital deaths from a TBI etiology (Marshall et al., 1991).

When studying the brain, lipids are of particular interest because they account for more than 50\% of its dry weight, are the major components of cell membranes, are repositories of chemical energy and play key roles in cell signaling and signal transduction (Fernandis and Wenk, 2007;Piomelli, 2005; Piomelli et al., 2007). The crucial role of lipids in brain physiology and cell signaling was demonstrated in neurologic disorders and several neurodegenerative diseases such as Alzheimer's, Parkinson's, and Niemann-Pick disease, in which the lipid metabolic pathways are disrupted (Sturley et al., 2004;Sharon et al., 2003;Cutler et al., 2004;Wenk, 2005). We previously studied a mild blast TBI 
model (Woods et al., 2013) and discovered a regionally specific accumulation of the ganglioside GM2 which plays a major role in cell recognition and signaling in the hippocampus, thalamus and hypothalamus. These changes were accompanied by depletion of ceramides, suggesting that lipids participate in both function and structure of neural membranes, and that disruption of lipid metabolism might be a key event in the pathophysiology of TBI (Adibhatla et al., 2006).

Driven by advances in mass spectrometry, the field of lipidomics has grown rapidly in the last two decades (Wenk, 2005;Wenk, 2010). Direct tissue analysis/mass spectrometry imaging (MSI) allows both the characterization of lipid species and the mapping of their distribution in tissue (Jackson et al., 2005;Colsch and Woods, 2010;Delvolve et al., 2011). Matrix-assisted laser desorption/ionization mass spectrometry (MALDI-MS) is the most commonly used mass spectrometric technique for MSI (Woods and Jackson, 2006;Fernandez et al., 2011). Since MSI is an in situ method, there is no pre-analysis separation step needed before mass analysis. Although simple in concept, proper sample preparation is a key prerequisite for detecting analytes of interest by MSI. Recently, we developed a highly accurate and reproducible method for implanting silver nanoparticles (AgNPs) in tissue sections (Jackson et al., 2014). Stable and homogenous AgNPs matrix layers results in highly reproducible tissue implantation, thus yielding high quality images of the regional distribution of several lipid species in both heart and kidney tissue as well as in a mouse model of chronic alcohol abuse (Jackson et al., 2014;Muller et al., 2015;Roux et al., 2014). In the current study, a controlled cortical impact (CCI) injury model (Romine et al., 2014) in adult male rats were used to study the progression of the pathology resulting from TBI up to 7 days post injury. MSI with AgNPs was used to locate, analyze and study the changes in brain lipid composition at different time points. 
2.1. Animals: All procedures were performed under protocols approved by the Uniformed

Services University Animal Care and Use Committee. Adult male Sprague-Dawley rats, weighing 225-

$250 \mathrm{~g}$ at the start of the study, were kept in a 12/12 hr light/dark cycle with access to food and water ad

libitum. Animals were divided in 2 groups, controls (Sham) and Injured (CCI). The CCI group was

subdivided into 3 sub-groups, reflecting the time elapsed after trauma before the animals were sacrifice (at day 1, 3 and 7). All groups, including Sham, contained 4 animals.

2.2. Controlled cortical Impact: This method uses a rigid impactor to deliver mechanical energy

to intact dura, exposed following a craniotomy (Romine et al., 2014) in rats anesthetized with isoflurane.

The impact was centered $2.04 \mathrm{~mm}$ posterior to bregma and $3.0 \mathrm{~mm}$ left of the midline (Figure 1) [-2.04 mm, Figure 50 in Paxinos \& Watson rat brain atlas (Paxinos and Watson, 2007)]. The impactor had a 3 $\mathrm{mm}$ diameter flat tip and the parameters used for the impact were a depth of $2.0 \mathrm{~mm}$, a velocity of $5 \mathrm{~m} / \mathrm{s}$ and a latency of $2 \mathrm{~ms}$. Control animals received no cortical impact strike, but otherwise received the same treatment as the CCI animals, including a craniotomy under isoflurane anesthesia. The animals were euthanized 1, 3 or 7 day post injury. Under ketamine/xylazine (100 mg/kg; $10 \mathrm{mg} / \mathrm{kg})$ anesthesia, the chest of each rat was opened and the head perfused through a catheter placed in the ascending aorta with 50 to100 $\mathrm{ml}$ of phosphate buffered saline at room temperature, allowing blood to flush from the head through an opening in the superior vena cava. When the perfusate was largely clear of blood, the skull was carefully opened and the brain dissected. After removing meninges, each brain was rapidly frozen in a small beaker containing about $30 \mathrm{ml}$ of cold isopentane pre-cooled by immersion of the beaker in solid

$\mathrm{CO}_{2}$, then removed, wrapped individually in aluminum foil and stored at $-80^{\circ} \mathrm{C}$ until sectioned. Coronal $18 \mu \mathrm{m}$ sections through the area of the injury were cut using a cryostat (Leica Microsystems CM3050S, 


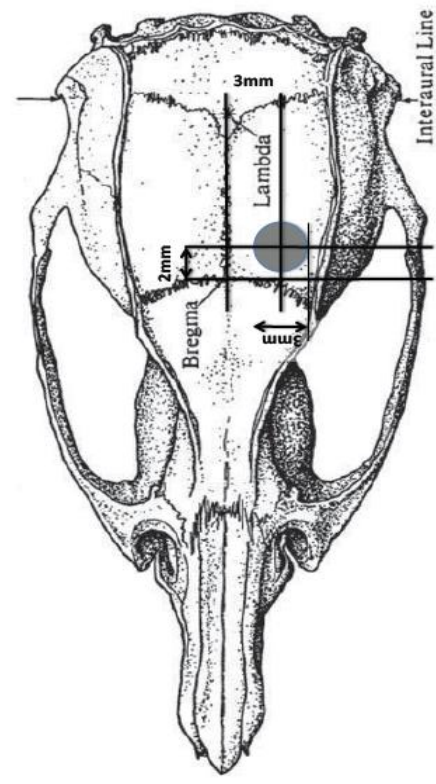

129

\section{Location of the controlled cortical Impact (CCI): $2.04 \mathrm{~mm}$ posterior to Bregma $3.0 \mathrm{~mm}$ left of Lambda}



Control brain

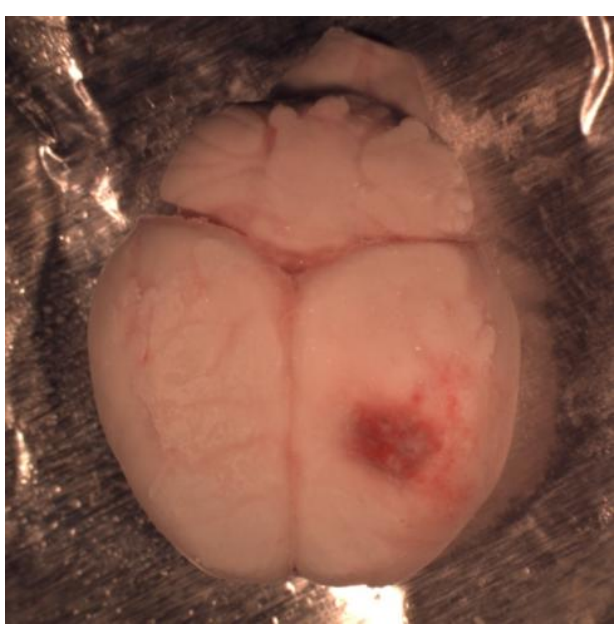

CCI brain (3 days)

Figure 1: Location of the Controlled cortical Impact in the Adult male Sprague-Dawley rat brains.

2.3. Tissue implantation and imaging: Tissue sections were implanted with silver nanoparticles (AgNP) $6 \mathrm{~nm}$ in diameter, using a nanoparticle implanter (Ionwerks, Houston, TX). A Thermo Scientific MALDI LTQ-XL-Orbitrap (Thermo Fisher Scientific, San Jose, CA) and Xcalibur software were used for MALDI mass spectrometry imaging (MSI) data acquisition. MS images of coronal sections near the level of bregma $-2.04 \mathrm{~mm}$ were collected in positive and negative ion mode. The mass resolution was set to 60,000 for positive ion mode and 30,000 for negative ion mode and the mass ranges were 630-950 Da and 650-1000 Da respectively. The raster step size was $50 \mu \mathrm{m}$ for both the $\mathrm{X}$ and $\mathrm{Y}$ directions. Imaging data were further analyzed with ImageWerks software (Ionwerks, Houston, TX). Images for a specific lipid are displayed using the same linear color scale to allow a visual comparison of intensities between samples.

Intensities in positive ion mode were not normalized, but intensities in negative ion were normalized using the intensity of all ions in the spectrum to compensate for instrumental analytical variability (samples in 
142 negative ion mode were analyzed over a longer period of time). MS/MS was performed to confirm each 143 lipid structure using CID energy between15 and 40\% but not all MS/MS were conclusive (Table 2).

2.4. Data Processing \& Statistical analysis: To investigate changes in the injured area, regions of 145 interest (ROI) delineating the injury were hand drawn for each lipid image using the free draw option of 146 the ImageWerks software. The ROI sizes are reported in pixels, with a pixel corresponding to the raster 147 step of the laser $(50 \mu \mathrm{m})$ and an area of $2500 \mu \mathrm{m}^{2}$. The ROIs for each lipid were initially determined as the 148 region surrounding the center of the impact site that showed the greatest change in levels of the specific 149 lipid in sections from animals subjected to CCI. The ROIs were similar for each lipid family but 150 considerably different between lipid families (i.e., SMs showed a different post-injury distribution from 151 CERs; see Results section). The average intensity/pixel of mass resolved lipid ions within each defined 152 ROI was computed by integrating the total intensity within the ROI and dividing by the number of pixels. 153 Resulting peak lists ( $\mathrm{m} / \mathrm{z}$ and intensities) were annotated using our lipid database. A maximum error of 5 154 parts-per-million (ppm) was used for the annotation. The ROI size and average intensities were compared 155 between animal groups and time points. The intensity of all detected lipids in each sample/ROI were 156 investigated by univariate analysis (two-way ANOVA and Bonferroni post-hoc tests) using GraphPad 157 Prism (version 4.00 for Windows, GraphPad Software, San Diego California USA). 


\section{Results}

160

161

162

163

164

165

3.1. Lipid Assignments: MSI analysis was conducted in both positive and negative ion mode in the injured coronal level (bregma -2.04 mm). MS/MS was performed on every annotated lipid. Most MS/MS were conclusive for lipid structure assignments; results of structural analysis are shown in Table 2. In positive ion mode, 41 lipids were detected as $[\mathrm{M}+\mathrm{Ag}]^{+}$ions, except for cholesterol that was detected as $\left.\left[\mathrm{M}+\mathrm{Ag}_{3}\right]^{+}\right)$. Although several other peaks associated with sodium and potassium adducts were identified, they were not used in this study as the natural distributions of sodium and potassium in tissue can be different from the lipid specie of interest. Previous imaging studies in stroke have shown that sodium adducts of lipids are greatly increased due to the presence of blood leading to erroneous results in which a lipid species appears to increase in the injury, although the increase is due to the amount of sodium in the injured area (Wang et al., 2010;Wang et al., 2012). Because interfering signals were present in some cases for the $\left[\mathrm{M}+{ }^{107} \mathrm{Ag}\right]^{+}$isotopic peak, the $\left[\mathrm{M}+{ }^{109} \mathrm{Ag}\right]^{+}$peak was used for intensity extraction as the ${ }^{107} \mathrm{Ag}$ and ${ }^{109} \mathrm{Ag}$ isotopes have similar abundance (51.84\% and $48.16 \%$ respectively). Four lipids had interferences for both $\mathrm{Ag}$ isotopes and were not analyzed. As a result 37 identified lipids were imaged in positive ion mode, including 4 ceramides (CER), 9 diacylglycerols (DAG), 8 cholesteryl esters (CE), 13 galactosyl ceramides (GALCER), 2 phosphatidylcholines (PC) and cholesterol (CHL). Of the 13 identified GALCER positive ion species, 7 were hydroxylated. Fifty-six lipids were detected as $[\mathrm{M}-\mathrm{H}]^{-}$ ions in negative ion mode, except for sphingomyelins which were detected as $\left[\mathrm{M}-\mathrm{H}-\mathrm{CH}_{2}\right]^{-}$. All 56 detected lipids were imaged with no interferences, including 8 sphingomyelins (SM), 19 phosphatidylethanolamines (PE), 6 phosphatidylinositols (PI) and 23 sulfatides (ST). Of the 23 ST imaged, 11 were hydroxylated. The 93 lipids imaged in this study and are listed in Table 2.

3.2. Characterization of CCI Injury by MSI of Lipid Species: The images for each lipid were compared across groups (control vs. injury for the different time points). Lipids from all classes (except 
182 GalCER and ST) show changes in the injury for at least one time point compared to controls. Some

183 changes were intense enough to clearly discern the injured area from the rest of the brain. Hence, the ROIs

184 were defined by pixels that differ significantly from the control concentrations of regional telencephalic

185 distribution of the same species. Hence, regions of interest (ROI) were defined using the lipid distribution

186 in the injured area for species that allowed a good delineation of the injury area in pixels. The ROIs were

187 found to be very similar within lipid species (e.g. all DAG show the same distribution in the injury) but

188 often differed between classes of lipid (e.g. SM have a different distribution than CER, Figure 2A). DAG

189 and SM showed similar distribution at 3 days (Figure 2A), however ST and GALCER did not show any

190 distinctive distribution within the injury making it difficult to define a ROI.

The average size in pixels ( $50 \mu \mathrm{m} \times 50 \mu \mathrm{m}$ sampling intervals) of each of the injury ROI at the 3

different time points were compared to controls (injury size $=0$ ) (Figure 2B and Table 1). One day after

the injury, the only lipid family to show a significant, focal increase in concentrations around the CCI site

was CER, suggesting that increased CER is an early biomarker of intracranial tissue reaction to primary

injury. At 3 days CER, CE, SM and DAG species show a significant injury size, suggesting that most of

the lipid changes occur by day 3 post injury. At seven days post-injury, the injured area's size decreased

for CER, CE and SM, whereas DAG completely disappeared from the injured area. The average ion count

of each lipid inside the ROI defining their distribution was also investigated to evaluate the intensity of the changes within the injured area at the different time points. Average intensity/pixel of mass resolved lipid ions correspond to the total intensity within the ROI and dividing by the number of pixels of the ROI. The

ROI for each lipid family determined in sections from injured brains was overlaid on the sections from

Sham rats, so that changes in intensity in injured animals could be compared to the intensities observed in the sham-injured brains. ROI were defined for CER, DAG, CE and SM, but as previously mentioned, 

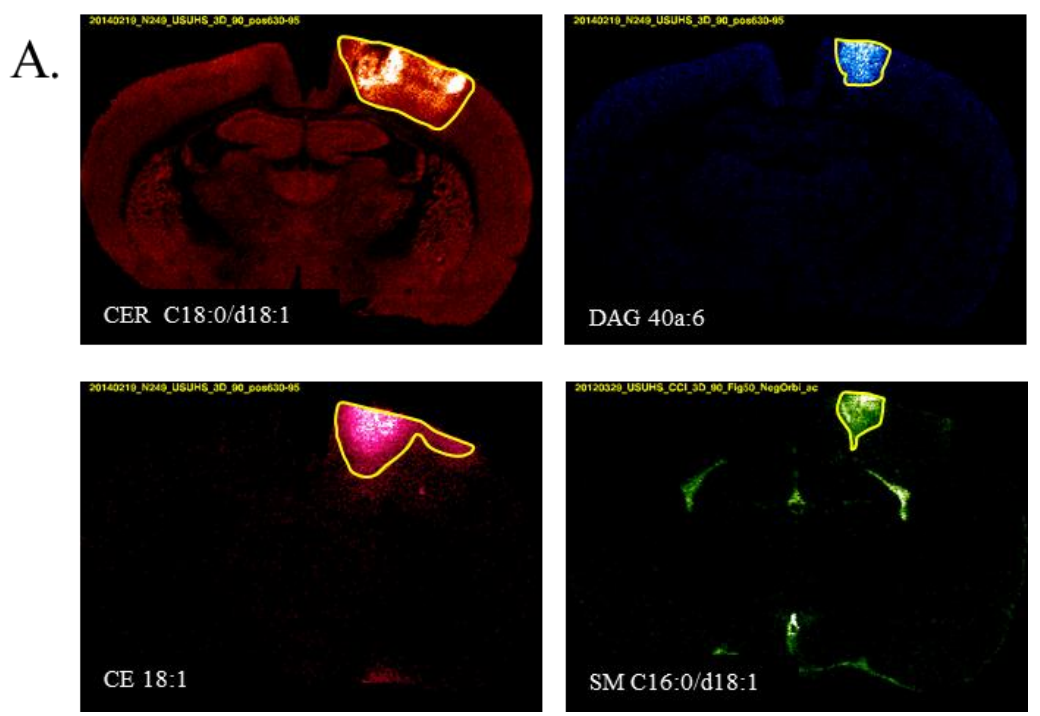

B. Injury size over time

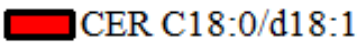

CE 18:1

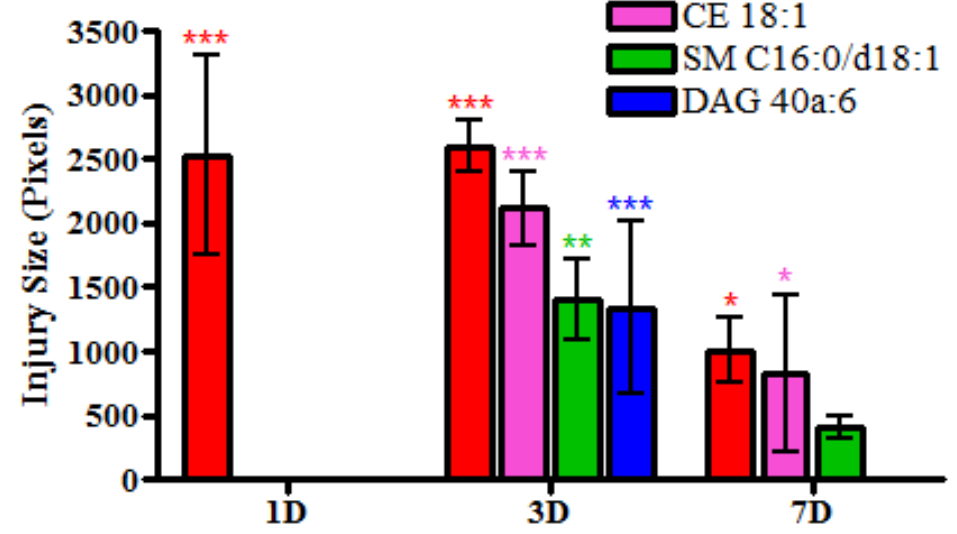

208 Figure 2: Size of the different ROIs defining the area of injury region for CER C18:0/d18:1 (m/z 674.45, 209 red), CE 18:1 (m/z 757.51, pink), SM C16:0/d18:1 (m/z 687.54, green) and DAG 40a:6 (m/z 775.44, 210 blue). A. MSI at 3 days for the lipids that were used to define the injury ROI. B. Graphs of the size of the 211 injury ROI over time (Bonferroni post-test compared to controls injury size $=0, * \mathrm{p}<0.05, * * \mathrm{p}<0.01, * * *$ $212 \mathrm{p}<0.001)$. When no changes were observed at a specific time point, no ROI was defined as for CE 18:1, 213 SM C16:0/d18:1 and DAG 40a:6 1 day after the injury. 
215 Table 1: Average size of the different ROIs defining the injury region for CER 674, DAG 775 CE 757 \&

216 SM 687. Results of the ANOVA and Bonferroni post-test compared to controls injury size $=0 .(\mathrm{AVG}=$

217 average in pixel $50 \mu \mathrm{m} * 50 \mu \mathrm{m})$

\begin{tabular}{|c|c|c|c|c|c|c|c|c|c|c|}
\hline Name (Mode) & $m / z$ & Lipid Classes & \multicolumn{2}{|c|}{$\begin{array}{l}\text { ANOVA } \\
\text { p-value }\end{array}$} & \multicolumn{6}{|c|}{ Bonferroni's Multiple Comparison Test } \\
\hline CER $674(+)$ & 674.4481 & CER/GALCER/PC & $P<0.0001$ & $* * *$ & $2527 \pm 782$ & $\mathrm{P}<0.001 * * *$ & $2596 \pm 200$ & $\mathrm{P}<0.001 * * *$ & $1003 \pm 253$ & $\mathrm{P}<0.05^{*}$ \\
\hline CE $757(+)$ & 757.5052 & $\mathrm{CE} / \mathrm{CHL}$ & $P<0.0001$ & $* * *$ & 0 & $\mathrm{P}>0.05$ & $2115 \pm 288$ & $\mathrm{P}<0.001 * * *$ & $820 \pm 615$ & $\mathrm{P}<0.05^{*}$ \\
\hline SM $687(-)$ & 687.5446 & $\mathrm{SM} / \mathrm{ST} / \mathrm{PE} / \mathrm{PI}$ & 0.0019 & $* *$ & 0 & $\mathrm{P}>0.05$ & $1410 \pm 632$ & $\mathrm{P}<0.01 * *$ & $401 \pm 158$ & $\mathrm{P}>0.05$ \\
\hline
\end{tabular}

AVG in pixel $=50 \mu \mathrm{m} * 50 \mu \mathrm{m}$

Another approach to visualizing spatial differences in lipid responses at the cortical impact site is shown in Figures 3-6, which illustrate the size and location of samples with CER, CE, and DAG levels

221 that exceeded the $99.5^{\text {th }}$ percentile observed across samples from the sections of four sham control

222 animals. The areas containing these high valued samples in the vicinity of the CCI site showed significant 223 size differences for different lipid species on days 1, 3 and 7 (Figure 3). CER C18:0/d18:1 was elevated 224 over a relatively large area on day 1; the area of elevated levels did not change on day 3, but dropped 225 significantly on day 7 (least significant differences test, $\mathrm{p}<0.05)$. Areas with high levels of CE (18:1), 226 DAG 40a:6 (18:0/22:6) and CER C16:0/d18:1 all showed a similar pattern: a significant increase on day 2273 , follow by a significant drop on day 7 (least significant differences tests, $\mathrm{p}<0.05)$. PC 34:1 (18:1/16:0)

228 showed a steady increase but the area was small. 


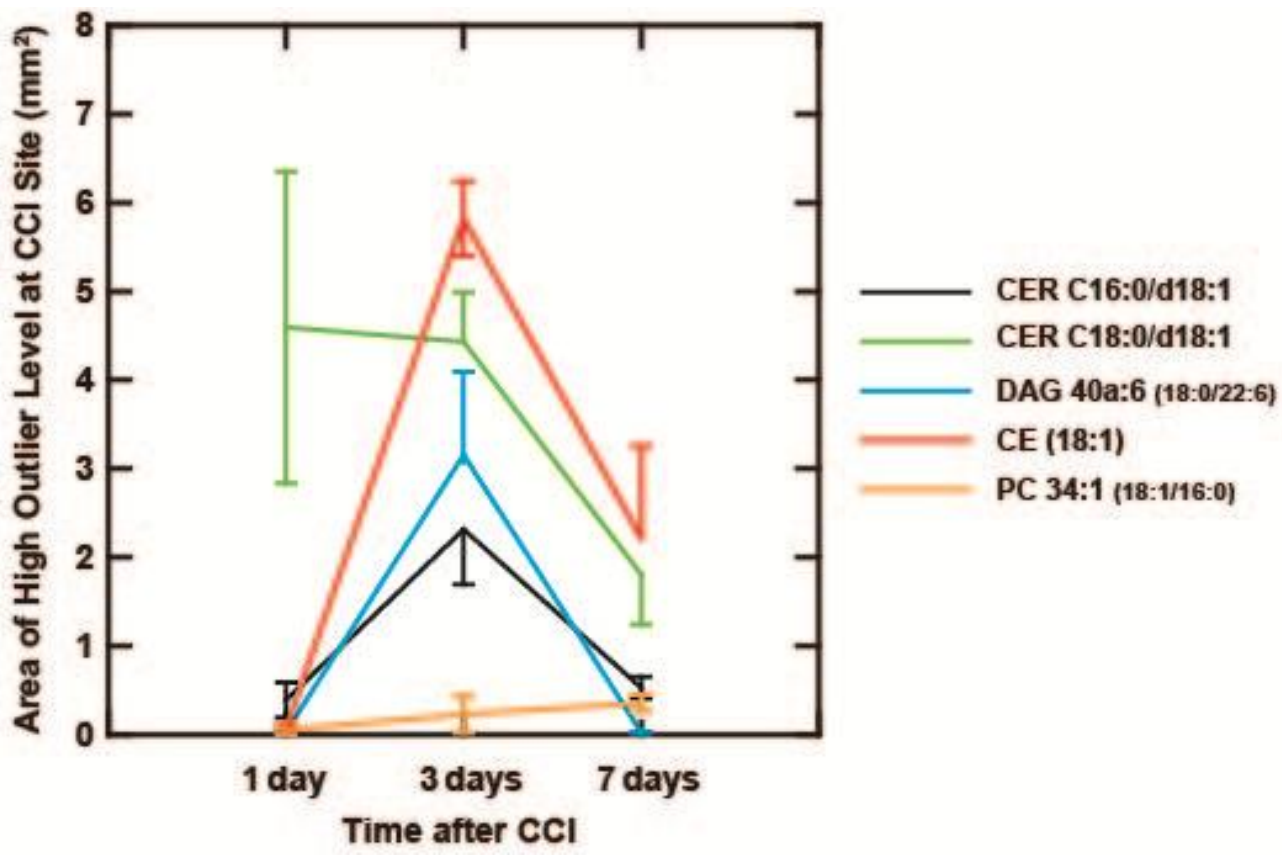

Figure 3. The area occupied by $50 \mu \mathrm{m}$ samples that showed levels greater than the $99.5^{\text {th }}$

231 percentile of sham control brains for five lipid species. The levels were measured at the total area under 232 the MS peaks for each positive ion species (m/z 644.4171 CER C16:0/d18:1[M+Ag107] ${ }^{+}, \mathrm{m} / \mathrm{z} 674.4481$ 233 CER C18:0/d18:1 [Ag109] [M+Ag109] ${ }^{+}, \mathrm{m} / \mathrm{z}$ 775.4430 DAG 40a:6 (18:0/22:6) [M+Ag107] $]^{+}$m/z $234757.5052 \mathrm{CE}(18: 1)[\mathrm{M}+\mathrm{Ag} 107]^{+}$, and m/z 866.4824 PC 34:1 (18:1/16:0) $\left.[\mathrm{M}+\mathrm{Ag} 107]^{+}\right)$, and the 99.5 $5^{\text {th }}$ 235 percentile point was identified from more than 200,000 pooled sampling sites from sections from 4 sham 236 control rats (anesthesia and craniotomy but no CCI). The spatial distributions of sites showing lipid 237 concentrations greater than the respective $99.5^{\text {th }}$ percentile cutoff at the CCI site are shown in Figures 4-6. 238 The number of $50 \mu \mathrm{m}$ sites was divided by 400 to yield the area of high outlier levels this graph; error bars 239 represent the standard error. The relative distribution of the areas showing high $\left(>99.5^{\text {th }}\right.$ percentile of control) values of these 241 lipids is compared for several positive ion species from each animal in Figures 4 (day 1), 5 (day 3 ) and 7 242 (day 7). On day 1, the high levels of CER C18:0/d18:1 dominated the CCI site and 91\% of the high CER 243 C16:0/d18:1 sites also had high levels of CER C18:0/d108:1 (Figure 4). The few sample sites with high 
244 levels of CE (18:1) and DAG 40a:6 (18:0/22:6) did not overlap appreciably with the former species. At 3 245 days after CCI, the spatial distribution of samples showing highly elevated values of CER C16:0/d18:1, 246 CE (18:1) and DAG 40a:6 (18:0/22:6) increased dramatically, such that the four species showed extensive 247 mutual overlap in single samples (Figure 5). At 7 days after CCI, the reduced areas of elevated CER 248 C18:0/d108:1, CER C16:0/d18:1 and CE (18:1) concentrations still showed extensive mutual overlap 249 (Figure 6), but there were virtually no sites of elevated DAG 40a:6 (18:0/22:6).
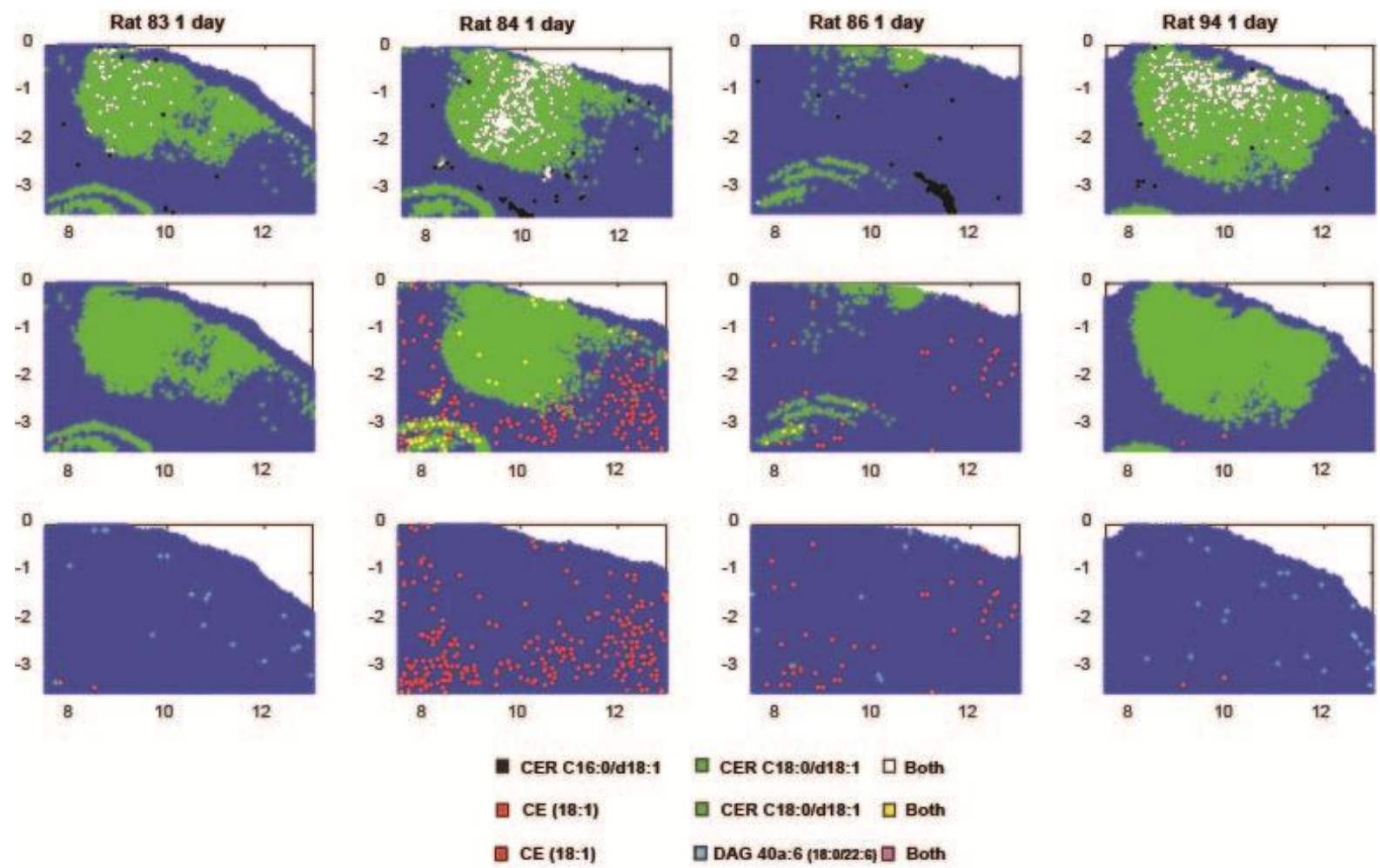

Figure 4. The distribution $50 \mu \mathrm{m}$ samples that showed levels greater than the 99.5 th percentile of sham 252 control brains for four lipid species, one day after CCI. The levels were measured at the total area under 253 the MS peaks for each positive ion species (m/z 644.4171 CER C16:0/d18:1[M+Ag107]+, m/z 674.4481 254 CER C18:0/d18:1 [Ag109] [M+Ag109]+, m/z 775.4430 DAG 40a:6 (18:0/22:6) [M+Ag107]+, m/z $255757.5052 \mathrm{CE}(18: 1)[\mathrm{M}+\mathrm{Ag} 107]+$, and m/z 866.4824 PC 34:1 (18:1/16:0) [M+Ag107]+). The data are 256 displayed from a section through the CCI site in four rats. The upper row shows data for sample sites 
257 displaying high values of the $\mathrm{CER}^{16}$ alone, $\mathrm{CER}^{18}$ alone and both. The middle row shows data for 258 sample sites displaying high values of the cholesteryl ester $(\mathrm{CE})$ alone, $C E R \mathrm{C}^{18}$ alone and both. The 259 lower row shows the distributions for outlier levels of CE alone, outlier levels of the DAG species alone and both in the same sample.
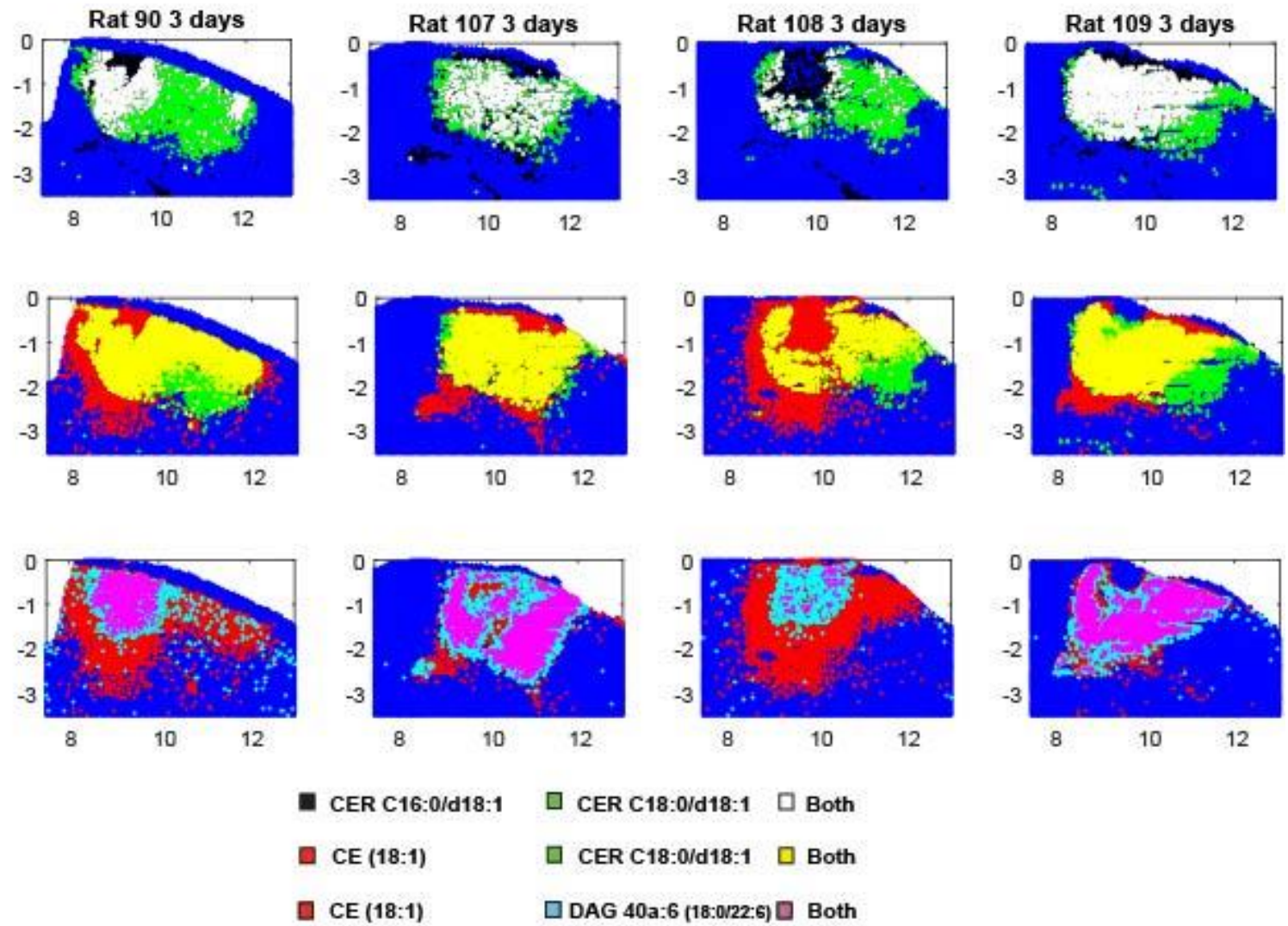

Figure 5. The distribution $50 \mu \mathrm{m}$ samples that showed levels greater than the 99.5 th percentile of sham control brains for four lipid species, three days after CCI. The levels were measured at the total area under the MS peaks for each positive ion species (m/z 644.4171 CER C16:0/d18:1[M+Ag107]+, m/z 674.4481 CER C18:0/d18:1 [Ag109] [M+Ag109]+, m/z 775.4430 DAG 40a:6 (18:0/22:6) [M+Ag107]+, m/z 757.5052 CE (18:1) [M+Ag107]+, and m/z 866.4824 PC 34:1 (18:1/16:0) [M+Ag107]+). The data are 267 displayed from a section through the CCI site in four rats. The upper row shows data for sample sites 268 displaying high values of the $\mathrm{CER}^{16}$ alone, $\mathrm{CER}^{18}$ alone and both. The middle row shows data for 
sample sites displaying high values of the cholesteryl ester (CE) alone, $C E R \mathrm{C}^{18}$ alone and both. The

270 lower row shows the distributions for outlier levels of CE alone, outlier levels of the DAG species alone 271 and both in the same sample.
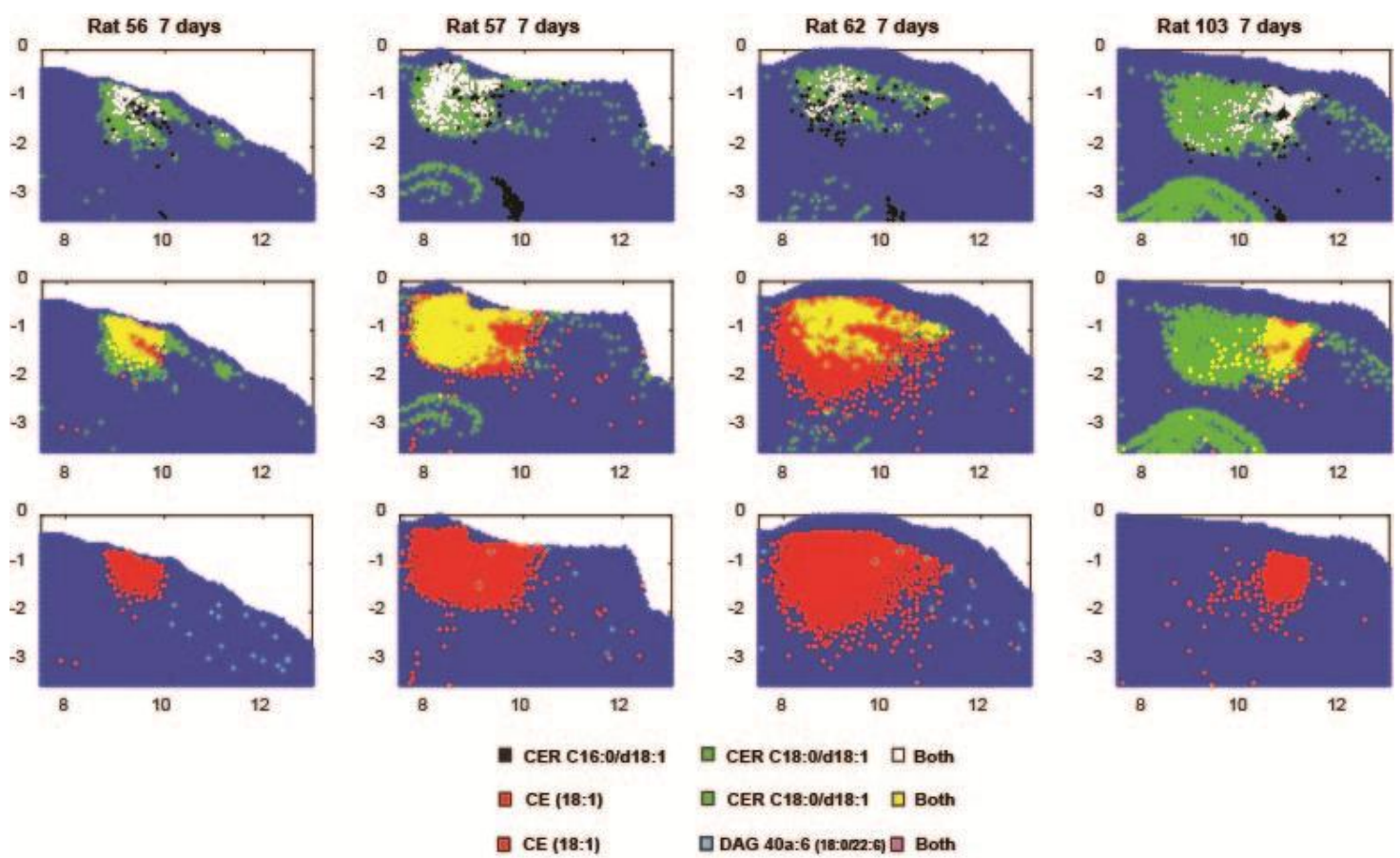

272

273 Figure 6. The distribution $50 \mu \mathrm{m}$ samples that showed levels greater than the 99.5 th percentile of sham control brains for four lipid species, seven days after CCI. The levels were measured at the total area 275 under the MS peaks for each positive ion species (m/z 644.4171 CER C16:0/d18:1[M+Ag107]+, m/z 674.4481 CER C18:0/d18:1 [Ag109] [M+Ag109]+, m/z 775.4430 DAG 40a:6 (18:0/22:6) [M+Ag107]+, m/z 757.5052 CE (18:1) [M+Ag107]+, and m/z 866.4824 PC 34:1 (18:1/16:0) [M+Ag107]+). The data 278 are displayed from a section through the CCI site in four rats. The upper row shows data for sample sites 279 displaying high values of the $\mathrm{CER} \mathrm{C}^{16}$ alone, $\mathrm{CER} \mathrm{C}^{18}$ alone and both. The middle row shows data for 280 sample sites displaying high values of the cholesteryl ester (CE) alone, CER $\mathrm{C}^{18}$ alone and both. The 281 lower row shows the distributions for outlier levels of CE alone, outlier levels of the DAG species alone 282 and both in the same sample. 
GALCER and ST did not show any change in the injured area, so no ROI could be drawn.

284 GALCER are glycosphingolipids with a ceramide backbone, therefore the same ROI that was used for 285 CER in positive ion mode was used for GALCER. ST are sulfated galactocerebrosides with a sphingosine 286 backbone, therefore the same ROI that was used for SM in negative ion mode was used for ST.

287 Glycerophospholipids PC, PE and PI exhibited minimal changes in the injury, they mostly decreased, 288 making it difficult to define a ROI. In positive ion mode, the PC distribution was found to be identical to 289 CER so the CER ROI was used for PC analysis. In negative ion mode, PE and PI distributions were 290 identical to SM so the SM ROI was used for these lipids. Results for average ion count changes were 291 grouped by lipid families (Table 2) including sphingolipids (CER, SM, GALCER \& ST), 292 glycerolipids/glycerophospholipids (PC, PI, PE \& DAG), cholesterol and its derivatives (CE) and are 293 discussed below. 


\begin{tabular}{|c|c|c|c|c|c|c|c|c|c|c|c|}
\hline \multirow{2}{*}{ Name } & \multirow{2}{*}{$m / z$} & \multirow{2}{*}{ Ion } & \multirow{2}{*}{$\begin{array}{c}\text { ANOVA } \\
\text { p-value }\end{array}$} & \multicolumn{8}{|c|}{ Bonferroni's Multiple Comparison Test } \\
\hline & & & & Sham vs $1 \mathrm{D}$ & & Sham vs 3D & & Sham vs 7D & $1 \mathrm{D}$ vs $3 \mathrm{D}$ & $1 \mathrm{D}$ vs $7 \mathrm{D}$ & $3 \mathrm{D}$ vs $7 \mathrm{D}$ \\
\hline CER C16:0/d18:1† & 644.4171 & {$\left[\mathrm{M}+\mathrm{Ag}_{107}\right]^{+}$} & $0.0036 * *$ & $>0.05$ & & $<0.01 * *$ & $\uparrow$ & $>0.05$ & $<0.05^{*}$ & $>0.05$ & $>0.05$ \\
\hline CER C18:1/d18:1† & 670.4328 & {$\left[\mathrm{M}+\mathrm{Ag}_{107}\right]^{+}$} & $0.0006 * * *$ & $<0.001 * * *$ & $\uparrow$ & $<0.05^{*}$ & $\uparrow$ & $>0.05$ & $>0.05$ & $>0.05$ & $>0.05$ \\
\hline CER C18:0/d18:1†[ $\left[\mathrm{Ag}_{109}\right]$ & 674.4481 & {$\left[\mathrm{M}+\mathrm{Ag}_{109}\right]^{+}$} & $0.045^{* *}$ & $<0.01 * *$ & $\uparrow$ & $<0.05^{*}$ & $\uparrow$ & $>0.05$ & $>0.05$ & $>0.05$ & $>0.05$ \\
\hline CER C20:0/d18: $1 \dagger$ & 700.4797 & {$\left[\mathrm{M}+\mathrm{Ag}_{107}\right]^{+}$} & 0.0139* & $<0.05^{*}$ & $\uparrow$ & $<0.05^{*}$ & $\uparrow$ & $>0.05$ & $>0.05$ & $>0.05$ & $>0.05$ \\
\hline $\mathrm{SM} \mathrm{C16:0/d18:1 \dagger}$ & 687.5446 & {$\left[\mathrm{M}-\mathrm{H}-\mathrm{CH}_{2}\right]^{-}$} & $0.0032 * *$ & $>0.05$ & & $<0.01 * *$ & $\uparrow$ & $>0.05$ & $<0.01 * *$ & $>0.05$ & $<0.05^{*}$ \\
\hline SM C18:1/d18:1† & 713.5603 & {$\left[\mathrm{M}-\mathrm{H}-\mathrm{CH}_{2}\right]^{-}$} & $0.0059 * *$ & $>0.05$ & & $>0.05$ & & $>0.05$ & $>0.05$ & $<0.05^{*}$ & $<0.01 * *$ \\
\hline SM C18:0/d18:1† & 715.5759 & {$\left[\mathrm{M}-\mathrm{H}-\mathrm{CH}_{2}\right]^{-}$} & 0.055 & $>0.05$ & & $>0.05$ & & $>0.05$ & $>0.05$ & $>0.05$ & $>0.05$ \\
\hline SM C20:1/d18:1 or C20:0/d18:2 & 741.5916 & {$\left[\mathrm{M}-\mathrm{H}-\mathrm{CH}_{2}\right]^{-}$} & na & na & & na & & na & na & na & na \\
\hline SM C20:0/d18:1 & 743.6072 & {$\left[\mathrm{M}-\mathrm{H}-\mathrm{CH}_{2}\right]^{-}$} & $0.0285^{*}$ & $>0.05$ & & $>0.05$ & & $>0.05$ & $>0.05$ & $>0.05$ & $>0.05$ \\
\hline SM C22:1/d18:1 or C22:0/d18:2 & 769.6229 & {$\left[\mathrm{M}-\mathrm{H}-\mathrm{CH}_{2}\right]^{-}$} & na & na & & na & & na & na & na & na \\
\hline SM C22:0/d18:1 & 771.6385 & {$\left[\mathrm{M}-\mathrm{H}-\mathrm{CH}_{2}\right]^{-}$} & 0.2166 & $>0.05$ & & $>0.05$ & & $>0.05$ & $>0.05$ & $>0.05$ & $>0.05$ \\
\hline SM C24:1/d18:1 or C24:0/d18:2 & 797.6542 & {$\left[\mathrm{M}-\mathrm{H}-\mathrm{CH}_{2}\right]^{-}$} & $0.0404 *$ & $>0.05$ & & $>0.05$ & & $>0.05$ & $>0.05$ & $>0.05$ & $>0.05$ \\
\hline GalCER C18:0/d18:1† & 834.5013 & {$\left[\mathrm{M}+\mathrm{Ag}_{107}\right]^{+}$} & 0.6186 & $>0.05$ & & $>0.05$ & & $>0.05$ & $>0.05$ & $>0.05$ & $>0.05$ \\
\hline GalCER C20:0/d18:1† & 862.5326 & {$\left[\mathrm{M}+\mathrm{Ag}_{107}\right]^{+}$} & 0.8651 & $>0.05$ & & $>0.05$ & & $>0.05$ & $>0.05$ & $>0.05$ & $>0.05$ \\
\hline GalCER C20:0/d18:1(OH)† & 878.5275 & {$\left[\mathrm{M}+\mathrm{Ag}_{107}\right]^{+}$} & 0.7091 & $>0.05$ & & $>0.05$ & & $>0.05$ & $>0.05$ & $>0.05$ & $>0.05$ \\
\hline GalCER C22:1/d18:1† & 888.5482 & {$\left[\mathrm{M}+\mathrm{Ag}_{107}\right]^{+}$} & 0.2249 & $>0.05$ & & $>0.05$ & & $>0.05$ & $>0.05$ & $>0.05$ & $>0.05$ \\
\hline GalCER C22:0/d18:1† [Ag109] & 892.5635 & {$\left[\mathrm{M}+\mathrm{Ag}_{109}\right]^{+}$} & 0.1710 & $>0.05$ & & $>0.05$ & & $>0.05$ & $>0.05$ & $>0.05$ & $>0.05$ \\
\hline GalCER C22:1/d18:1(OH)† & 904.5431 & {$\left[\mathrm{M}+\mathrm{Ag}_{107}\right]^{+}$} & 0.1048 & $>0.05$ & & $>0.05$ & & $>0.05$ & $>0.05$ & $>0.05$ & $>0.05$ \\
\hline GalCER C22:0/d18:1(OH) $\dagger\left[\mathrm{Ag}_{109}\right]$ & 908.5584 & {$\left[\mathrm{M}+\mathrm{Ag}_{109}\right]^{+}$} & $0.0413 *$ & $>0.05$ & & $>0.05$ & & $>0.05$ & $>0.05$ & $>0.05$ & $>0.05$ \\
\hline GalCER C24:1/d18:2 \& C24:2/d18:1† & 914.5639 & {$\left[\mathrm{M}+\mathrm{Ag}_{107}\right]^{+}$} & 0.2463 & $>0.05$ & & $>0.05$ & & $>0.05$ & $>0.05$ & $>0.05$ & $>0.05$ \\
\hline GalCER C24:1/d18:1† & 916.5795 & {$\left[\mathrm{M}+\mathrm{Ag}_{107}\right]^{+}$} & 0.087 & $>0.05$ & & $>0.05$ & & $>0.05$ & $>0.05$ & $>0.05$ & $>0.05$ \\
\hline GalCER C23:0/d18:1(OH)† $\left[\mathrm{Ag}_{109}\right]$ & 922.5741 & {$\left[\mathrm{M}+\mathrm{Ag}_{109}\right]^{+}$} & 0.2445 & $>0.05$ & & $>0.05$ & & $>0.05$ & $>0.05$ & $>0.05$ & $>0.05$ \\
\hline GalCER C24:1/d18:2(OH)† & 930.5588 & {$\left[\mathrm{M}+\mathrm{Ag}_{107}\right]^{+}$} & 0.5032 & $>0.05$ & & $>0.05$ & & $>0.05$ & $>0.05$ & $>0.05$ & $>0.05$ \\
\hline GalCER C24:1/d18:1(OH)† & 932.5744 & {$\left[\mathrm{M}+\mathrm{Ag}_{107}\right]^{+}$} & 0.1812 & $>0.05$ & & $>0.05$ & & $>0.05$ & $>0.05$ & $>0.05$ & $>0.05$ \\
\hline GalCER C24:0/d18:1(OH)† [Ag $\left.\mathrm{Ag}_{109}\right]$ & 936.5897 & {$\left[\mathrm{M}+\mathrm{Ag}_{109}\right]^{+}$} & 0.0533 & $>0.05$ & & $>0.05$ & & $>0.05$ & $>0.05$ & $>0.05$ & $>0.05$ \\
\hline ST C16:1/d18:1 or C16:0/d18:2 & 776.4988 & {$[\mathrm{M}-\mathrm{H}]^{-}$} & 0.1704 & $>0.05$ & & $>0.05$ & & $>0.05$ & $>0.05$ & $>0.05$ & $>0.05$ \\
\hline ST C18:1/d18:2 & 802.5145 & {$[\mathrm{M}-\mathrm{H}]^{-}$} & 0.1642 & $>0.05$ & & $>0.05$ & & $>0.05$ & $>0.05$ & $>0.05$ & $>0.05$ \\
\hline ST C18:1/d18:1 or C18:0/d18:2 & 804.5301 & {$[\mathrm{M}-\mathrm{H}]^{-}$} & 0.7272 & $>0.05$ & & $>0.05$ & & $>0.05$ & $>0.05$ & $>0.05$ & $>0.05$ \\
\hline ST C18:0/d18:1 & 806.5458 & {$[\mathrm{M}-\mathrm{H}]^{-}$} & 0.3758 & $>0.05$ & & $>0.05$ & & $>0.05$ & $>0.05$ & $>0.05$ & $>0.05$ \\
\hline ST C18:1/d18:1 or C18:0/d18:2(OH) & 820.5250 & {$[\mathrm{M}-\mathrm{H}]^{-}$} & 0.3899 & $>0.05$ & & $>0.05$ & & $>0.05$ & $>0.05$ & $>0.05$ & $>0.05$ \\
\hline ST C18:0/d18:1(OH)† & 822.5407 & {$[\mathrm{M}-\mathrm{H}]^{-}$} & 0.1296 & $>0.05$ & & $>0.05$ & & $>0.05$ & $>0.05$ & $>0.05$ & $>0.05$ \\
\hline ST C20:1/d $18: 1$ or C20:0/d18:2 & 832.5614 & {$[\mathrm{M}-\mathrm{H}]^{-}$} & 0.9619 & $>0.05$ & & $>0.05$ & & $>0.05$ & $>0.05$ & $>0.05$ & $>0.05$ \\
\hline ST C20:0/d18:1 & 834.5771 & {$[\mathrm{M}-\mathrm{H}]^{-}$} & 0.8750 & $>0.05$ & & $>0.05$ & & $>0.05$ & $>0.05$ & $>0.05$ & $>0.05$ \\
\hline ST C20:1/d18:1 or C20:0/d18:2(OH) & 848.5563 & {$[\mathrm{M}-\mathrm{H}]^{-}$} & 0.9047 & $>0.05$ & & $>0.05$ & & $>0.05$ & $>0.05$ & $>0.05$ & $>0.05$ \\
\hline ST C20:0/d18:1(OH) & 850.5720 & {$[\mathrm{M}-\mathrm{H}]^{-}$} & 0.6148 & $>0.05$ & & $>0.05$ & & $>0.05$ & $>0.05$ & $>0.05$ & $>0.05$ \\
\hline ST C22:1/d18:1 or C22:0/d18:2 & 860.5927 & {$[\mathrm{M}-\mathrm{H}]^{-}$} & 0.8938 & $>0.05$ & & $>0.05$ & & $>0.05$ & $>0.05$ & $>0.05$ & $>0.05$ \\
\hline
\end{tabular}




\begin{tabular}{|c|c|c|c|c|c|c|c|c|c|c|}
\hline ST C22:0/d18:1† & 862.6084 & {$[\mathrm{M}-\mathrm{H}]^{-}$} & 0.3535 & $>0.05$ & $>0.05$ & & $>0.05$ & $>0.05$ & $>0.05$ & $>0.05$ \\
\hline ST C22:0/d18:1(OH) $\dagger$ & 878.6033 & {$[\mathrm{M}-\mathrm{H}]^{-}$} & 0.5479 & $>0.05$ & $>0.05$ & & $>0.05$ & $>0.05$ & $>0.05$ & $>0.05$ \\
\hline ST C24:1/d18:2 & 886.6084 & {$[\mathrm{M}-\mathrm{H}]^{-}$} & 0.5351 & $>0.05$ & $>0.05$ & & $>0.05$ & $>0.05$ & $>0.05$ & $>0.05$ \\
\hline ST C24:1/d18:1† & 888.6240 & {$[\mathrm{M}-\mathrm{H}]^{-}$} & $0.0209 *$ & $>0.05$ & $>0.05$ & & $<0.05^{*}$ & $>0.05$ & $>0.05$ & $>0.05$ \\
\hline ST C24:0/d18:1† & 890.6397 & {$[\mathrm{M}-\mathrm{H}]^{-}$} & 0.2542 & $>0.05$ & $>0.05$ & & $>0.05$ & $>0.05$ & $>0.05$ & $>0.05$ \\
\hline ST C24:1/d18:2(OH) & 902.6033 & {$[\mathrm{M}-\mathrm{H}]^{-}$} & 0.9884 & $>0.05$ & $>0.05$ & & $>0.05$ & $>0.05$ & $>0.05$ & $>0.05$ \\
\hline ST C24:1/d18:1(OH) $\dagger$ & 904.6189 & {$[\mathrm{M}-\mathrm{H}]^{-}$} & 0.2126 & $>0.05$ & $>0.05$ & & $>0.05$ & $>0.05$ & $>0.05$ & $>0.05$ \\
\hline ST C24:0/d18:1(OH) $\dagger$ & 906.6346 & {$[\mathrm{M}-\mathrm{H}]^{-}$} & 0.4702 & $>0.05$ & $>0.05$ & & $>0.05$ & $>0.05$ & $>0.05$ & $>0.05$ \\
\hline ST C26:1/d18:1 or C26:0/d18:2 & 916.6553 & {$[\mathrm{M}-\mathrm{H}]^{-}$} & 0.7257 & $>0.05$ & $>0.05$ & & $>0.05$ & $>0.05$ & $>0.05$ & $>0.05$ \\
\hline ST C25:0/d18:1(OH) $\dagger$ & 920.6502 & {$[\mathrm{M}-\mathrm{H}]^{-}$} & 0.1958 & $>0.05$ & $>0.05$ & & $>0.05$ & $>0.05$ & $>0.05$ & $>0.05$ \\
\hline ST C26:1/d18:1 or C26:0/d18:2(OH) & 932.6502 & {$[\mathrm{M}-\mathrm{H}]^{-}$} & 0.4993 & $>0.05$ & $>0.05$ & & $>0.05$ & $>0.05$ & $>0.05$ & $>0.05$ \\
\hline ST C26:0/d18:1(OH) & 934.6659 & {$[\mathrm{M}-\mathrm{H}]^{-}$} & 0.3846 & $>0.05$ & $>0.05$ & & $>0.05$ & $>0.05$ & $>0.05$ & $>0.05$ \\
\hline DAG 32a:0 & 675.4117 & {$\left[\mathrm{M}+\mathrm{Ag}_{107}\right]^{+}$} & 0.3495 & $>0.05$ & $>0.05$ & & $>0.05$ & $>0.05$ & $>0.05$ & $>0.05$ \\
\hline DAG 34a:2 & 699.4117 & {$\left[\mathrm{M}+\mathrm{Ag}_{107}\right]^{+}$} & $0.0372 *$ & $>0.05$ & $>0.05$ & & $>0.05$ & $>0.05$ & $>0.05$ & $>0.05$ \\
\hline DAG 34a:1 $(16: 0 / 18: 1) \dagger$ & 701.4274 & {$\left[\mathrm{M}+\mathrm{Ag}_{107}\right]^{+}$} & 0.2731 & $>0.05$ & $>0.05$ & & $>0.05$ & $>0.05$ & $>0.05$ & $>0.05$ \\
\hline DAG 36a:4 & 723.4117 & {$\left[\mathrm{M}+\mathrm{Ag}_{107}\right]^{+}$} & $0.0029 * *$ & $>0.05$ & $<0.01 * *$ & $\uparrow$ & $>0.05$ & $<0.05^{*}$ & $>0.05$ & $<0.05^{*}$ \\
\hline DAG 36a:2 $(18: 1 / 18: 1+18: 0 / 18: 2) \dagger$ & 727.4430 & {$\left[\mathrm{M}+\mathrm{Ag}_{107}\right]^{+}$} & $<0.0001 * * *$ & $>0.05$ & $<0.001 * * *$ & $\uparrow$ & $>0.05$ & $<0.001 * * *$ & $>0.05$ & $<0.001 * * *$ \\
\hline DAG 36a:1 $\left[\mathrm{Ag}_{109}\right]$ & 731.4583 & {$\left[\mathrm{M}+\mathrm{Ag}_{109}\right]^{+}$} & $<0.0001 * * *$ & $>0.05$ & $<0.001 * *$ & $\uparrow$ & $>0.05$ & $<0.001 * *$ & $>0.05$ & $<0.001 * *$ \\
\hline DAG 38a:6 (20:6/18:0+22:6/16:0)† & 747.4117 & {$\left[\mathrm{M}+\mathrm{Ag}_{107}\right]^{+}$} & $<0.0001 * * *$ & $>0.05$ & $<0.001 * * *$ & $\uparrow$ & $>0.05$ & $<0.001 * * *$ & $>0.05$ & $<0.001 * * *$ \\
\hline DAG 38a:4 $(18: 0 / 20: 4) \dagger\left[\mathrm{Ag}_{109}\right]$ & 753.4427 & {$\left[\mathrm{M}+\mathrm{Ag}_{109}\right]^{+}$} & $<0.0001 * * *$ & $>0.05$ & $<0.001 * *$ & $\uparrow$ & $>0.05$ & $<0.001 * *$ & $>0.05$ & $<0.001 * *$ \\
\hline DAG 40a:6 (18:0/22:6)† & 775.4430 & {$\left[\mathrm{M}+\mathrm{Ag}_{107}\right]^{+}$} & $<0.0001 * * *$ & $>0.05$ & $<0.001 * * *$ & $\uparrow$ & $>0.05$ & $<0.001 * * *$ & $>0.05$ & $<0.001 * * *$ \\
\hline PE 32a:0 & 690.5080 & {$[\mathrm{M}-\mathrm{H}]^{-}$} & 0.3443 & $>0.05$ & $>0.05$ & & $>0.05$ & $>0.05$ & $>0.05$ & $>0.05$ \\
\hline PE 34a:1 $(16: 0 / 18: 1) \dagger$ & 716.5236 & {$[\mathrm{M}-\mathrm{H}]^{-}$} & $0.0012 * *$ & $>0.05$ & $<0.05^{*}$ & $\downarrow$ & $>0.05$ & $<0.01 * *$ & $>0.05$ & $<0.05^{*}$ \\
\hline PE 34a:0 & 718.5393 & {$[\mathrm{M}-\mathrm{H}]^{-}$} & 0.6265 & $>0.05$ & $>0.05$ & & $>0.05$ & $>0.05$ & $>0.05$ & $>0.05$ \\
\hline PE 36p:4 (16:0p/20:4)† & 722.5131 & {$[\mathrm{M}-\mathrm{H}]^{-}$} & 0.2023 & $>0.05$ & $>0.05$ & & $>0.05$ & $>0.05$ & $>0.05$ & $>0.05$ \\
\hline PE 36a:4 (16:0/20:4)† & 738.5080 & {$[\mathrm{M}-\mathrm{H}]^{-}$} & 0.3447 & $>0.05$ & $>0.05$ & & $>0.05$ & $>0.05$ & $>0.05$ & $>0.05$ \\
\hline PE 36a:3 $(18: 2 / 18: 1) \dagger$ & 740.5236 & {$[\mathrm{M}-\mathrm{H}]^{-}$} & 0.1387 & $>0.05$ & $>0.05$ & & $>0.05$ & $>0.05$ & $>0.05$ & $>0.05$ \\
\hline PE 36a:2 (18:1/18:1)† & 742.5393 & {$[\mathrm{M}-\mathrm{H}]^{-}$} & 0.0233* & $>0.05$ & $>0.05$ & & $>0.05$ & $>0.05$ & $<0.05^{*}$ & $>0.05$ \\
\hline PE 36a:1 $(18: 0 / 18: 1) \dagger$ & 744.5549 & {$[\mathrm{M}-\mathrm{H}]^{-}$} & 0.3001 & $>0.05$ & $>0.05$ & & $>0.05$ & $>0.05$ & $>0.05$ & $>0.05$ \\
\hline PE 38p:6 (16:0p/22:6)† & 746.5131 & {$[\mathrm{M}-\mathrm{H}]^{-}$} & 0.1358 & $>0.05$ & $>0.05$ & & $>0.05$ & $>0.05$ & $>0.05$ & $>0.05$ \\
\hline PE 36a:0 & 746.5706 & {$[\mathrm{M}-\mathrm{H}]^{-}$} & 0.0513 & $>0.05$ & $>0.05$ & & $>0.05$ & $>0.05$ & $>0.05$ & $>0.05$ \\
\hline PE 38a:6 (16:0/22:6)† & 762.5080 & {$[\mathrm{M}-\mathrm{H}]^{-}$} & $0.0095 * *$ & $>0.05$ & $<0.01 * *$ & $\downarrow$ & $>0.05$ & $>0.05$ & $>0.05$ & $>0.05$ \\
\hline PE 38a:5 (18:1/20:4)† & 764.5236 & {$[\mathrm{M}-\mathrm{H}]^{-}$} & 0.1727 & $>0.05$ & $>0.05$ & & $>0.05$ & $>0.05$ & $>0.05$ & $>0.05$ \\
\hline PE 38a:4 (18:0/20:4)† & 766.5393 & {$[\mathrm{M}-\mathrm{H}]^{-}$} & 0.8649 & $>0.05$ & $>0.05$ & & $>0.05$ & $>0.05$ & $>0.05$ & $>0.05$ \\
\hline PE 38a:2 & 770.5706 & {$[\mathrm{M}-\mathrm{H}]^{-}$} & 0.1391 & $>0.05$ & $>0.05$ & & $>0.05$ & $>0.05$ & $>0.05$ & $>0.05$ \\
\hline PE 38a:1 (18:1/20:0)† & 772.5862 & {$[\mathrm{M}-\mathrm{H}]^{-}$} & 0.1387 & $>0.05$ & $>0.05$ & & $>0.05$ & $>0.05$ & $>0.05$ & $>0.05$ \\
\hline PE 40p:6 (18:0p/22:6)† & 774.5444 & {$[\mathrm{M}-\mathrm{H}]^{-}$} & 0.1485 & $>0.05$ & $>0.05$ & & $>0.05$ & $>0.05$ & $>0.05$ & $>0.05$ \\
\hline PE 40a:6 (18:0/22:6)† & 790.5393 & {$[\mathrm{M}-\mathrm{H}]^{-}$} & $0.0170 * *$ & $>0.05$ & $<0.05^{*}$ & $\downarrow$ & $>0.05$ & $>0.05$ & $>0.05$ & $>0.05$ \\
\hline PE 40a:5 & 792.5549 & {$[\mathrm{M}-\mathrm{H}]^{-}$} & 0.2654 & $>0.05$ & $>0.05$ & & $>0.05$ & $>0.05$ & $>0.05$ & $>0.05$ \\
\hline PE 40a:4 & 794.5706 & {$[\mathrm{M}-\mathrm{H}]^{-}$} & 0.0585 & $>0.05$ & $>0.05$ & & $>0.05$ & $>0.05$ & $>0.05$ & $>0.05$ \\
\hline PC 32:0 $(16: 0 / 16: 0) \dagger$ & 840.4667 & {$\left[\mathrm{M}+\mathrm{Ag}_{107}\right]^{+}$} & 0.0801 & $>0.05$ & $>0.05$ & & $>0.05$ & $>0.05$ & $>0.05$ & $>0.05$ \\
\hline PC 34:1 (18:1/16:0)† & 866.4824 & {$\left[\mathrm{M}+\mathrm{Ag}_{107}\right]^{+}$} & $0.0077 * *$ & $>0.05$ & $>0.05$ & & $>0.05$ & $>0.05$ & $<0.05^{*}$ & $<0.05^{*}$ \\
\hline
\end{tabular}




\begin{tabular}{|c|c|c|c|c|c|c|c|c|c|c|c|}
\hline PI 34:1 & 835.5342 & {$[\mathrm{M}-\mathrm{H}]^{-}$} & 0.3458 & $>0.05$ & $>0.05$ & & $>0.05$ & & $>0.05$ & $>0.05$ & $>0.05$ \\
\hline PI 36:4 (16:0/20:4 )† & 857.5186 & {$[\mathrm{M}-\mathrm{H}]^{-}$} & $0.0151 *$ & $>0.05$ & $>0.05$ & & $>0.05$ & & $>0.05$ & $>0.05$ & $<0.05^{*}$ \\
\hline PI 38:6 (18:0/20:4 \& 16:0/22:4)† & 881.5186 & {$[\mathrm{M}-\mathrm{H}]^{-}$} & 0.3615 & $>0.05$ & $>0.05$ & & $>0.05$ & & $>0.05$ & $>0.05$ & $>0.05$ \\
\hline PI 38:5 (18:1/20:4)† & 883.5342 & {$[\mathrm{M}-\mathrm{H}]^{-}$} & 0.1508 & $>0.05$ & $>0.05$ & & $>0.05$ & & $>0.05$ & $>0.05$ & $>0.05$ \\
\hline PI 38:4 (18:0/20:4)† & 885.5499 & {$[\mathrm{M}-\mathrm{H}]^{-}$} & 0.2508 & $>0.05$ & $>0.05$ & & $>0.05$ & & $>0.05$ & $>0.05$ & $>0.05$ \\
\hline PI 40:6 (18:0/22:6)† & 909.5499 & {$[\mathrm{M}-\mathrm{H}]^{-}$} & 0.7023 & $>0.05$ & $>0.05$ & & $>0.05$ & & $>0.05$ & $>0.05$ & $>0.05$ \\
\hline Cholesterol $\left[\mathrm{Ag}_{109}\right]$ & 709.0698 & {$\left[\mathrm{M}+\mathrm{Ag}_{3}\right]^{+}$} & 0.6396 & $>0.05$ & $>0.05$ & & $>0.05$ & & $>0.05$ & $>0.05$ & $>0.05$ \\
\hline CE (16:0) & 731.4896 & {$\left[\mathrm{M}+\mathrm{Ag}_{107}\right]^{+}$} & $<0.0001 * * *$ & $>0.05$ & $<0.001 * * *$ & $\uparrow$ & $<0.001 * * *$ & $\uparrow$ & $<0.001 * * *$ & $<0.001 * * *$ & $<0.05^{*}$ \\
\hline CE (18:2) & 755.4896 & {$\left[\mathrm{M}+\mathrm{Ag}_{107}\right]^{+}$} & $0.0078 * *$ & $>0.05$ & $<0.05^{*}$ & $\uparrow$ & $>0.05$ & & $<0.05^{*}$ & $>0.05$ & $<0.05^{*}$ \\
\hline $\mathrm{CE}(18: 1) \dagger$ & 757.5052 & {$\left[\mathrm{M}+\mathrm{Ag}_{107}\right]^{+}$} & $0.0011 * *$ & $>0.05$ & $<0.01 * *$ & $\uparrow$ & $<0.05^{*}$ & $\uparrow$ & $<0.01 * *$ & $<0.05^{*}$ & $>0.05$ \\
\hline $\mathrm{CE}(20: 4) \dagger\left[\mathrm{Ag}_{109}\right]$ & 781.4892 & {$\left[\mathrm{M}+\mathrm{Ag}_{109}\right]^{+}$} & $<0.0001 * * *$ & $>0.05$ & $<0.001 * * *$ & $\uparrow$ & $<0.001 * * *$ & $\uparrow$ & $<0.001 * * *$ & $<0.01^{* *}$ & $>0.05$ \\
\hline $\operatorname{CE}(22: 6) \dagger$ & 803.4896 & {$\left[\mathrm{M}+\mathrm{Ag}_{107}\right]^{+}$} & $<0.0001 * * *$ & $>0.05$ & $<0.01 * *$ & $\uparrow$ & $<0.001 * * *$ & $\uparrow$ & $<0.01 * *$ & $<0.001 * * *$ & $>0.05$ \\
\hline $\mathrm{CE}(22: 4)\left[\mathrm{Ag}_{109}\right]$ & 809.5205 & {$\left[\mathrm{M}+\mathrm{Ag}_{109}\right]^{+}$} & $0.0010 * *$ & $>0.05$ & $<0.05^{*}$ & $\uparrow$ & $<0.05^{*}$ & $\uparrow$ & $<0.05^{*}$ & $<0.05^{*}$ & $>0.05$ \\
\hline CE (24:6) & 831.5209 & {$\left[\mathrm{M}+\mathrm{Ag}_{107}\right]^{+}$} & $0.0004 * * *$ & $>0.05$ & $>0.05$ & & $<0.001 * * *$ & $\uparrow$ & $>0.05$ & $<0.001 * * *$ & $<0.05^{*}$ \\
\hline CE $(24: 4)$ & 835.5522 & {$\left[\mathrm{M}+\mathrm{Ag}_{107}\right]^{+}$} & $<0.0001 * * *$ & $>0.05$ & $>0.05$ & & $<0.001 * * *$ & $\uparrow$ & $>0.05$ & $<0.001 * * *$ & $<0.01 * *$ \\
\hline
\end{tabular}

295 na=Below detection limits $(0.1 \%)$ in most of the samples, not enough data to do statistics. $\uparrow$ Structure confirmed my MS/MS 
3.3. Sphingolipids: ST and GALCER average intensities within the injury site did not show any

significant change between control and CCI animals. However CER and SM exhibited important changes as seen in Figure 7. The changes correlated to the fatty acid attached to the sphingoid base. Low mass SM and CER $\left(\leq \mathrm{C}_{16}\right)$ were localized exclusively in the ventricles in controls and in the injured area in CCI animals. Among low mass SM and CER, only SM \& CER C16:0/d18:1 were detected. They are probably derived from blood in the injury and thus are located in the hematoma region (Figure 7A). 3 CER and 4 $\mathrm{SM}$ of intermediate mass (from $\mathrm{C}_{18}$ to $\mathrm{C}_{20}$ ) were detected. They are localized in grey matter and show significant and opposite changes (increase in CER vs decrease in SM) in the injured area (Figure 7B). Only 3 high mass $\mathrm{SM}\left(\geq \mathrm{C}_{22}\right)$ were detected. They are localized in the ventricles and show no significant changes (data not shown) but a small increase in the injured area (which is an opposite trend compared to grey matter SM).

The intensities extracted from the CER and SM ROI, confirmed as low mass SM and CER C16:0/d18:1 (Figure 7A), were increased in the region of the injured area. Both SM and CER showed a significant increase in intensity after 3 days (Figure 7C) but no significant increase was observed for the other time-points, unlike for grey matter CER (Figure 7B). Intermediate mass SM and CER (C18:0/d18:1 Figure 7B) were localized in the grey matter of the brain. These SM and CER showed opposite changes within the injury. CER increased significantly as early as $24 \mathrm{~h}$ post trauma and remained elevated after 3 and 7 days although not significantly elevated at 7 days). In contrast, SM decreased in the injury after 1 and 3 days but this decrease was not significant (Figure 7D). High mass SM $\left(\geq C_{22}\right)$ localized in the ventricles showed no significant changes (data not shown). Since the injury was located in the cortex which is mainly grey matter, white matter species had a low intensity and exhibited a large variation within the injury's ROI. Statistics are summarized in Table 2. In addition, alteration of the brain lipid content was not limited to the injury ROI, as some species changed in the rest of the brain. Grey matter 
CER increased in the caudate putamen, globus pallidus and the hippocampus (Figure 7B), while grey

A. Blood related CER/SM

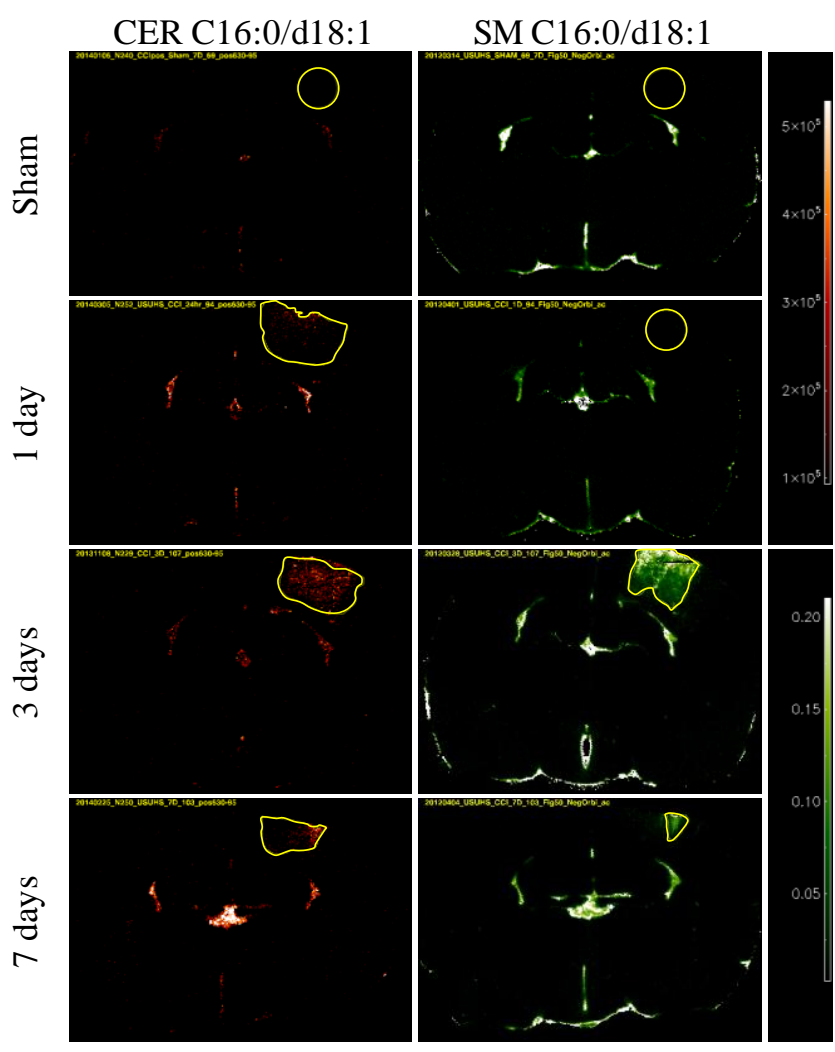

C. CER/SM C16:0/d18:1 average intensities

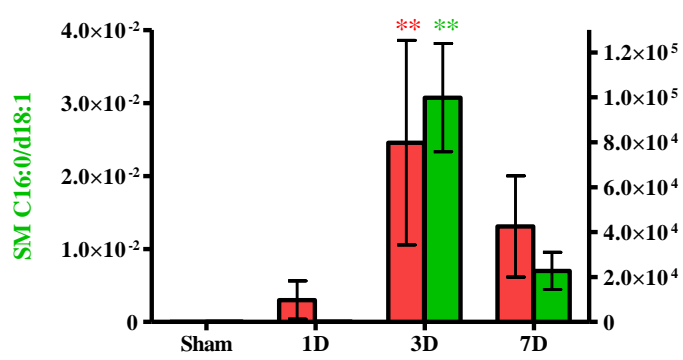

B. Grey Matter CER/SM



D. CER/SM C18:0/d18:1 average intensities



323 Figure 7: MSI of controls (Sham) and CCI brains (1 day, 3 days and 7 days) for blood related CER/SM

324 C16:0/d18:1 (A) and grey matter CER/SM C18:0/d18:1 (B) (CER in red, SM in green). Average 325 intensities within the injury ROI in controls (Sham) and CCI brains (1 day, 3 days and 7 days) for 326 CER/SM C16:0/d18:1 (C) and CER/SM C18:0/d18:1 (D) (CER in red, SM in green) (Bonferroni post-test 327 compared intensity in controls, $* \mathrm{p}<0.05, * * \mathrm{p}<0.01, * * * \mathrm{p}<0.001)$. 
(PC/PE/PI) mostly decreased in the injured area. All of the detected species in these 3 classes were predominantly localized in grey matter (Figure 8A \& B). DAG, a class of glycerolipids, was not detected 331 in control brain tissue (sham), but showed a marked increase in injured brains after 3 days (Figure 8A).

The average intensities extracted from the different ROI confirmed what was observed in the MS images in Figure 8. DAG showed a significant increase 3 days after the injury as is shown in Figure 8C. This 334 observed for the other detected glycerophospholipids. docosahexaenoic acid (DHA, FA 22:6) according to their MS/MS (16:0a/22:6 and 18:0/22:6 respectively; Table 1). For other glycerophospholipids classes (PI and PC), the 40a:6 diacyl backbone was not detected; PC34:1 (16:0/18:1) and PI 36:4 (16:0/20:4) are shown in Figure 8B. Both PC and PI showed a decrease at 3 days and an increase at 7 days. These changes were not significant compared to controls, but the decrease at 3 days was significant compared to the increase at 7 days (Figure 8D). In addition the PC showed a decrease at 1 day that was also significant compared to the increase at 7 days. Of the 2 PC and 6 PI detected, only PC34:1 and PI 36:4 exhibited significant changes even though a similar trend was 
A. DAG \& PE 40a:6

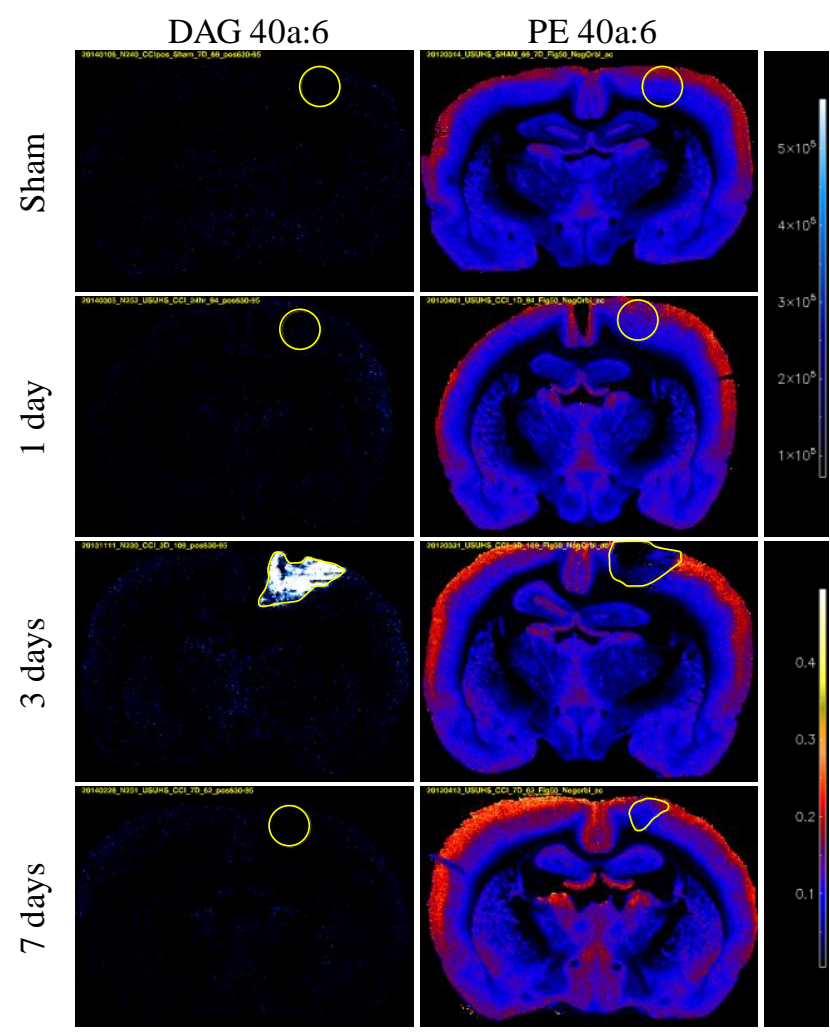

C. DAG \& PE 40a:6 average intensities

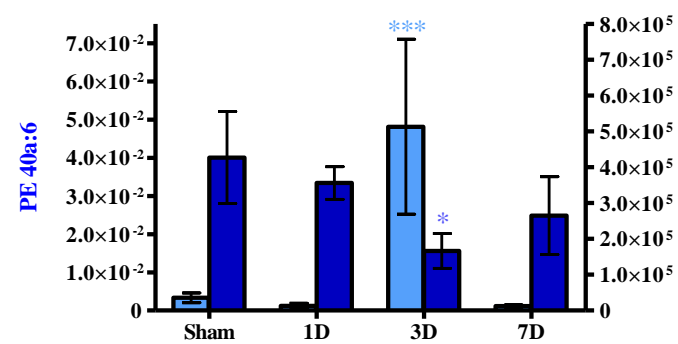

B. PC 34:1 \& PI 36:4



D. PC 34:1 \& PI 34:6 average intensities

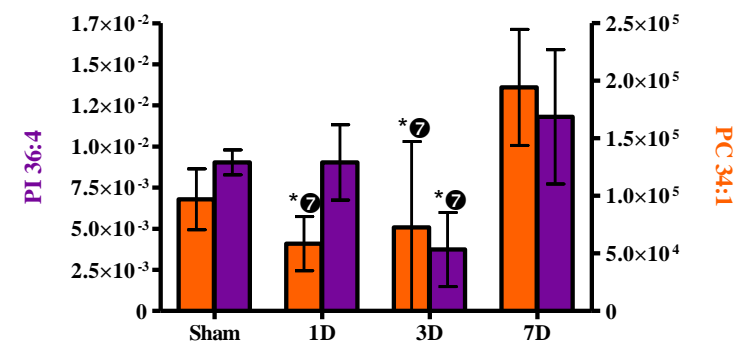

350 Figure 8: MSI of controls (Sham) and CCI brains (1 day, 3 days and 7 days) for DAG and PE 40a:6 (A),

351 PC 34:1 and PI 36:4 (B) (DAG in blue/white, PE in red/blue, PC and PI in rainbow). Average intensities

352 within the injury ROI in controls (Sham) and CCI brains (1 day, 3 days and 7 days) for DAG and PE

353 40a:6 (C), PC 34:1 and PI 36:4 (D) (DAG in light blue, PE in navy blue, PC in dark orange and PI in

354 purple). (Bonferroni post-test compared intensity in controls, $* \mathrm{p}<0.05, * * \mathrm{p}<0.01$, *** $\mathrm{p}<0.001$, for PC

355 and PI $\boldsymbol{7}$ means the significance is compared to 7 days, not sham). 
357 distribution in the injury area. Cholesterol was detected in the whole brain, and showed a decrease in the 358 injury region after 3 and 7 days (Figure 9A). It also showed an increase in the rest of the brain after 3

359 days, more specifically in the corpus callosum, dentate gyrus \& the internal capsule. CE were not detected 360 in control brains but increased in the injured area, and their signals were intense at the same time points 361 where cholesterol decreased ( $3 \& 7$ days). The average intensities extracted from the ROI confirmed the 362 significant increase of CE in the injury at 3 and 7 days (Figure 9B) but the cholesterol decrease observed 363 was not significant.



B. Cholesterol \& CE (22:6) average intensities



Figure 9: MSI of controls (Sham) and CCI brains (1 day, 3 days and 7 days) for cholesterol and CE 22:6 
367 brains (1 day, 3 days and 7 days) for cholesterol and CE 22:6 (CGL in light green, CE in light orange) (B).

368 (Bonferroni post-test compared intensity in controls, * $\mathrm{p}<0.05, * * \mathrm{p}<0.01, * * * \mathrm{p}<0.001$ ).

369

\section{Discussion}

Changes in lipid profile after CCI point to the magnitude of pathological changes and the biochemical pathways involved in both the secondary injury progression and mobilization of wound healing and repair mechanisms. At a molecular level, concussive brain injury is believed to produce neuronal injury and death by activating excitotoxic metabolic pathway, with one pathological process involving excessive stimulation of NMDA and AMPA receptors by the excitatory neurotransmitter glutamate (Olney, 1993;Olney J.W., 1978) and release of excessive amount of dynorphin (Woods et al., 2006; Hauser et al., 2005). Excitotoxicity is not specific to TBI; it is common to many neurological disorders including stroke, epilepsy, and neurodegenerative diseases (Waggie et al., 1999;Meldrum and Garthwaite, 1990). Cellular and molecular events induced by brain injury have been divided into three phases that involve both sequentially and temporally overlapping responses in the brain: initial and subsequent injuries and "repair process" (Levine et al., 2006). An excellent review by Hunt et al., 2013 has detailed the cascades from these three phases and the potential molecular and cellular events involved and will not be reiterated here (Hunt et al., 2013).

In this study, many lipid families showed changes over time at the site of CCI, including sphingolipids. Numerous papers and reviews have been published about the role of the SM/CER signaling pathway in cell death or apoptosis (Woodcock, 2006;Aureli et al., 2014; Taniguchi and Okazaki, 2014). Activation of this pathway occurs after cellular stress including ischemic brain injury and stroke (Altura et al., 2002; Yu et al., 2000;Novgorodov and Gudz, 2011). It results in the cleavage of membrane SM by activation of acidic and/or neutral sphingomyelinase (SMase) and generation of the second messenger ceramide. Our results suggest that the SM/CER signaling pathway is involved in TBI as we observed 
changes in both SM and CER. Both low mass SM and CER detected in this study (SM/CER C16:0/d18:1) showed a concomitant increase in the injured area. According to our MS images these low mass species SM \& CER $\mathrm{C}_{16}$ are not normally present in the brain parenchyma but they are usually found in blood (Gorska et al., 2002;Ichi et al., 2007;Ionescu et al., 2013). Their increase in the injury between day 1 and day 3, accompanied by increased CE, could indicate changes in blood-brain barrier properties, inflammatory cell migration, or an increased local synthesis rate. Intermediate mass SM and CER (from $\mathrm{C}_{18}$ to $\mathrm{C}_{20}$ ), localized in the gray matter, showed opposite trends 3 days after the injury: a decrease in SM and an increase in CER. These changes may reflect the activation of SMase that hydrolyzes SM to produce CER. However, the early increase of CER at day 1 is not correlated with a decrease in the corresponding SM, as observed at days 3. This suggests that the CER synthesis (de novo or salvage pathway with CER synthase CerS) might be activated as early as day1 post TBI. Indeed, a variety of stressful stimuli are known to increase CER levels via activation of both SMase and CerS (Stiban et al., 2010;Merrill, Jr. and Jones, 1990), especially CerS5 and CerS6 (Jin et al., 2008;Schull et al., 2015), which synthesize $\mathrm{C}_{14}-\mathrm{C}_{18} \mathrm{CER}$. This would also explain why CER are still increased in the injury after 7 days, while SM do not show any decrease because of no additional hydrolysis by SMase. Moreover the CER increase observed for intermediate mass SM and CER is not only localized in the injured area, but is also observed in other regions of the brain, suggesting the presence of diffuse axonal injury mediated most likely by activation of CerS or de novo synthesis since no corresponding SM decrease was observed in those regions. Finally, high mass SM $(\geq \mathrm{C} 22: 0)$, localized in the ventricles/white matter, seem to be less affected by events at the CCI site as no significant changes were observed.

Glycerolipids, glycerophospholipids, cholesterol and its derivatives also showed changes over time after TBI. DAGs are important second messenger signaling lipids produced from the degradation of glycerophospholipids by phospholipase C (PLC). It has been shown that brain injury increases the activity of PLC (Wei et al., 1982;Dhillon et al., 1999), releasing lipid second messengers including DAG. Our 
results suggest that the increase of DAG in the injury is most likely linked to the decrease/degradation of glycerophospholipids, including PE and probably PC and PI by an increase in PLC activity. Glycerophospholipids are major constituents of neural cell membranes particularly long chain polyunsaturated fatty acids (PUFA) such as arachidonic acid (AA; 20:4 n-6) and docosahexaenoic acid (DHA; 22:6 n-3). DHA is the most important PUFA PE in mature brain and interestingly, in this study, all PE containing either AA or DHA showed a trend toward a decrease in the injured area after 3 days (38p:6, 38a:6, 38a:5, 40p:6, 40a:6, 40a:5). However, only the diacyl-PE containing DHA were significantly decreased (38a:6 \& 40a:6). This result suggests membranes disruption. Cholesterol is another major component of the brain and neural cell membrane, $25 \%$ of the cholesterol in the body is found in the brain, where it plays important roles in membrane function. In this study a non-significant decrease of cholesterol was observed in the injury as well as a significant increase of CE which is produced by esterification of cholesterol. In tissues, including brain, the enzyme responsible for the esterification of free cholesterol is the acyl-coenzyme A cholesterol acyltransferase (ACAT). ACAT-mediated esterification of cholesterol limits the toxic accumulation of excess free cholesterol in cells (Tabas, 2002). ACAT1 expression was shown to increase after excitotoxic injury in the hippocampus, significantly increasing cholesteryl esters levels (Kim et al., 2011). The significant increase of CE observed at 3 and 7 days post injury could reflect similar local neurodegenerative events, possibly combined with vascular remodeling and secondary changes in blood-brain barrier permeability at the injury site.

Overall investigation of brain lipids at the site of CCI gives an insight into the extent of biochemical, functional and structural changes that may be detected by changes in lipid profiles. It is noteworthy that the spatial distributions of high levels of different CERs, CE, and DAG follow distinct time courses, suggesting that they are regulated independently by the local milieu (Figures 3-6). The lipid classes exhibiting the most significant changes in gray matter were CER, DAG and CE. The CER species increased as early as 1 day post injury and were still elevated after 7 days, possibly reflecting their role as 
438 signaling substrates, precursors for downstream synthesis of sphingomyelins, and turnover of

439 sphingomyelins, sphingosines, ceramide-1-phosphate and glucosylceramides. This behavior contrasts

440 sharply with decreased regional brain CER concentrations after low level blast wave exposure (Woods et

441 al., 2013), which, unlike CCI, produced no evidence of frank brain injury. Hence, we attribute the CER

442 response in this study to the injury and repair of a contusion of neural and vascular tissues.

443 The delayed elevation of CE is significant because levels are very low in normal brain, but may be

444 elevated in some neurodegenerative disorders (Dietschy and Turley, 2004). However, like the more

445 prolonged increase in $\mathrm{C}_{18} \mathrm{CER}$, the observed spatially overlapping $\mathrm{CE}$ increase is likely to be associated

446 with vascular remodeling and inflammatory reactions, as well as central nervous system neuronal and glial

447 responses. The effect at both 3 and 7 days post injury seems to be consistent with a role in multiple

448 wound healing and repair responses. The increase in DAG, on the other hand, appeared only at 3 days and

449 was associated spatially with sample sites showing high levels of blood-related $\mathrm{C}_{16}$ CER and CE. Hence,

450 it appears to be a more transient signal associated with tissue responses at a 'core' region of injury. Thus,

451 the ability to analyze quantitatively the spatio-temporal distribution of these lipid species in tissue sections

452 provide a new tool to investigate cellular processes of injury response and remodeling in neural, glial and

453 vascular components of injured brain tissue.

454 Acknowledgements: The authors declare no competing financial interests. This work was supported in part by 455 a grant from the Department of Defense and the Center for Neuroscience and Regenerative Medicine 456 (CNRM), Rockville, MD and by the Intramural Research Program of the National Institute on Drug Abuse, 457 NIH. Ionwerks gratefully acknowledges support of this work through NIH SBIR Phase II grants

458 R44DA030853-03 5R44DA036263-03". The authors acknowledge Dr. Mari Prieto and Glen Gregory of 459 the Thermo Fisher Corporation for technical and instrumentation advice. The authors also thank Gregory 460 Bull for his technical support. The opinions and assertions contained herein are the personal opinions of 
461 the authors and are not to be construed as official or reflecting the views of the Uniformed Services

462 University, the Department of Defense, NIDA or the NIH. 
Adibhatla RM, Hatcher JF, Dempsey RJ (2006) Lipids and lipidomics in brain injury and diseases. AAPS 467 J 8:E314-E321.

468 469

470

471

472

473

474

475

476

477

478

479

480

481

482

483

484

485

486

487

488

489

490

491

492

493

494

495

496

497
Agrawal A, Timothy J, Pandit L, Manju M (2006) Post-traumatic epilepsy: an overview. Clin Neurol Neurosurg 108:433-439.

Altura BM, Gebrewold A, Zheng T, Altura BT (2002) Sphingomyelinase and ceramide analogs induce vasoconstriction and leukocyte-endothelial interactions in cerebral venules in the intact rat brain: Insight into mechanisms and possible relation to brain injury and stroke. Brain Res Bull 58:271-278.

Aureli M, Murdica V, Loberto N, Samarani M, Prinetti A, Bassi R, Sonnino S (2014) Exploring the link between ceramide and ionizing radiation. Glycoconj J 31:449-459.

Colsch B, Woods AS (2010) Localization and imaging of sialylated glycosphingolipids in brain tissue sections by MALDI mass spectrometry. Glycobiology 20:661-667.

Cutler RG, Kelly J, Storie K, Pedersen WA, Tammara A, Hatanpaa K, Troncoso JC, Mattson MP (2004) Involvement of oxidative stress-induced abnormalities in ceramide and cholesterol metabolism in brain aging and Alzheimer's disease. Proc Natl Acad Sci U S A 101:2070-2075.

Delvolve AM, Colsch B, Woods AS (2011) Highlighting anatomical sub-structures in rat brain tissue using lipid imaging. Anal Methods 3:1729-1736.

Dhillon HS, Carman HM, Prasad RM (1999) Regional activities of phospholipase C after experimental brain injury in the rat. Neurochem Res 24:751-755.

Dietschy JM, Turley SD (2004) Thematic review series: brain Lipids. Cholesterol metabolism in the central nervous system during early development and in the mature animal. J Lipid Res 45:1375-1397.

Faul M, Xu L, Wald MM, Coronado VG (2010) Traumatic brain injury in the United States: emergency department visits, hospitalizations, and deaths. Atlanta (GA): Centers for Disease Control and Prevention, National Center for Injury Prevention and Control.

Fernandez JA, Ochoa B, Fresnedo O, Giralt MT, Rodriguez-Puertas R (2011) Matrix-assisted laser desorption ionization imaging mass spectrometry in lipidomics. Anal Bioanal Chem 401:29-51.

Fernandis AZ, Wenk MR (2007) Membrane lipids as signaling molecules. Curr Opin Lipidol 18:121-128.

Gorska M, Dobrzyn A, Zendzian-Piotrowska M, Namiot Z (2002) Concentration and composition of free ceramides in human plasma. Horm Metab Res 34:466-468.

Hardman JM, Manoukian A (2002) Pathology of head trauma. Neuroimaging Clin N Am 12:175-87, vii.

Hauser KF, Aldrich JV, Anderson KJ, Bakalkin G, Christie MJ, Hall ED, Knapp PE, Scheff SW, Singh IN, Vissel B, Woods AS, Yakovleva T, Shippenberg TS (2005) Pathobiology of dynorphins in trauma and disease. Front Biosci 10:216-235. 
Hunt RF, Boychuk JA, Smith BN (2013) Neural circuit mechanisms of post-traumatic epilepsy. Front Cell Neurosci 7:89.

Ichi I, Nakahara K, Kiso K, Kojo S (2007) Effect of dietary cholesterol and high fat on ceramide concentration in rat tissues. Nutrition 23:570-574.

Ionescu N, de FC, Bueno AA (2013) Perturbations in blood phosphatidylcholine and sphingomyelin Fatty Acid composition in a sample population of cigarette smokers. Indian J Clin Biochem 28:361-367.

Jackson SN, Baldwin K, Muller L, Womack VM, Schultz JA, Balaban C, Woods AS (2014) Imaging of lipids in rat heart by MALDI-MS with silver nanoparticles. Anal Bioanal Chem 406:1377-1386.

Jackson SN, Wang HY, Woods AS (2005) Direct profiling of lipid distribution in brain tissue using MALDI-TOFMS. Anal Chem 77:4523-4527.

Jin J, Hou Q, Mullen TD, Zeidan YH, Bielawski J, Kraveka JM, Bielawska A, Obeid LM, Hannun YA, Hsu YT (2008) Ceramide generated by sphingomyelin hydrolysis and the salvage pathway is involved in hypoxia/reoxygenation-induced Bax redistribution to mitochondria in NT-2 cells. J Biol Chem 283:2650926517.

Kim JH, Ee SM, Jittiwat J, Ong ES, Farooqui AA, Jenner AM, Ong WY (2011) Increased expression of acyl-coenzyme A: cholesterol acyltransferase-1 and elevated cholesteryl esters in the hippocampus after excitotoxic injury. Neuroscience 185:125-134.

Kushner D (1998) Mild traumatic brain injury: toward understanding manifestations and treatment. Arch Intern Med 158:1617-1624.

Levine B, Fujiwara E, O'Connor C, Richard N, Kovacevic N, Mandic M, Restagno A, Easdon C, Robertson IH, Graham SJ, Cheung G, Gao F, Schwartz ML, Black SE (2006) In vivo characterization of traumatic brain injury neuropathology with structural and functional neuroimaging. J Neurotrauma 23:1396-1411.

Marshall LF, Gautille T, Klauber MR, Eisenberg HM, Jane JA, Luerssen TG, Marmarou A, Foulkes MA (1991) The outcome of severe closed head injury. Special Supplements 75:S28-S36.

Meldrum B, Garthwaite J (1990) Excitatory amino acid neurotoxicity and neurodegenerative disease. Trends Pharmacol Sci 11:379-387.

Merrill AH, Jr., Jones DD (1990) An update of the enzymology and regulation of sphingomyelin metabolism. Biochim Biophys Acta 1044:1-12.

Muller L, Kailas A, Jackson SN, Roux A, Barbacci DC, Schultz JA, Balaban CD, Woods AS (2015) Lipid imaging within the normal rat kidney using silver nanoparticles by matrix-assisted laser desorption/ionization mass spectrometry. Kidney Int.

National Institute of Neurological Disorders and Stroke. (2002) Traumatic brain injury: hope through research. Bethesda (MD): National Institutes of Health.

Novgorodov SA, Gudz TI (2011) Ceramide and mitochondria in ischemic brain injury. Int J Biochem Mol Biol 2:347-361. 
534 Olney J.W. (1978) Neurotoxicity of excitatory amino acids. In: Kainic Acid as a Tool in Neurobiology 535 (McGeer E., Olney JW, McGeer P., eds), pp 95-121. New York: Raven Press.

536 Olney JW (1993) Role of excitotoxins in developmental neuropathology. APMIS Suppl 40:103-112.

537 Paxinos G, Watson C (2007) The Rat Brain in Stereotaxic Coordinates. Academic Press.

538 Piomelli D (2005) The challenge of brain lipidomics. Prostaglandins Other Lipid Mediat 77:23-34.

539 Piomelli D, Astarita G, Rapaka R (2007) A neuroscientist's guide to lipidomics. Nat Rev Neurosci 8:743540754.

541 Rao V, Lyketsos C (2000) Neuropsychiatric sequelae of traumatic brain injury. Psychosomatics 41:95542103.

543 Romine J, Gao X, Chen J (2014) Controlled cortical impact model for traumatic brain injury. J Vis 544 Expe51781.

545 Roux A, Muller L, Jackson SN, Baldwin K, Womack V, Pagiazitis JG, O'Rourke JR, Thanos PK, Balaban 546 C, Schultz JA, Volkow ND, Woods AS (2014) Chronic Ethanol Consumption Profoundly Alters Regional 547 Brain Ceramide and Sphingomyelin Content in Rodents. ACS Chem Neurosci 6:247-259.

548 Schull S, Gunther SD, Brodesser S, Seeger JM, Tosetti B, Wiegmann K, Pongratz C, Diaz F, Witt A, 549 Andree M, Brinkmann K, Kronke M, Wiesner RJ, Kashkar H (2015) Cytochrome c oxidase deficiency 550 accelerates mitochondrial apoptosis by activating ceramide synthase 6. Cell Death Dis 6:e1691.

551 Sharon R, Bar-Joseph I, Frosch MP, Walsh DM, Hamilton JA, Selkoe DJ (2003) The formation of highly 552 soluble oligomers of alpha-synuclein is regulated by fatty acids and enhanced in Parkinson's disease.

553 Neuron 37:583-595.

554 Stiban J, Tidhar R, Futerman AH (2010) Ceramide synthases: roles in cell physiology and signaling. Adv 555 Exp Med Biol 688:60-71.

556 Sturley SL, Patterson MC, Balch W, Liscum L (2004) The pathophysiology and mechanisms of NP-C 557 disease. Biochim Biophys Acta 1685:83-87.

558 Tabas I (2002) Consequences of cellular cholesterol accumulation: basic concepts and physiological 559 implications. J Clin Invest 110:905-911.

560

561

562

563

564

565

566

567

568
Taniguchi M, Okazaki T (2014) The role of sphingomyelin and sphingomyelin synthases in cell death, proliferation and migration-from cell and animal models to human disorders. Biochim Biophys Acta 1841:692-703.

Waggie KS, Kahle PJ, Tolwani RJ (1999) Neurons and mechanisms of neuronal death in neurodegenerative diseases: a brief review. Lab Anim Sci 49:358-362.

Wang HY, Liu CB, Wu HW, Kuo JS (2010) Direct profiling of phospholipids and lysophospholipids in rat brain sections after ischemic stroke. Rapid Commun Mass Spectrom 24:2057-2064.

Wang HY, Wu HW, Tsai PJ, Liu CB (2012) MALDI-mass spectrometry imaging of desalted rat brain sections reveals ischemia-mediated changes of lipids. Anal Bioanal Chem 404:113-124. 
569 Wei EP, Lamb RG, Kontos HA (1982) Increased phospholipase C activity after experimental brain injury.

570 J Neurosurg 56:695-698.

571 Wenk MR (2005) The emerging field of lipidomics. Nat Rev Drug Discov 4:594-610.

572 Wenk MR (2010) Lipidomics: new tools and applications. Cell 143:888-895.

573 Woodcock J (2006) Sphingosine and ceramide signalling in apoptosis. IUBMB Life 58:462-466.

574 Woods AS, Colsch B, Jackson SN, Post J, Baldwin K, Roux A, Hoffer B, Cox BM, Hoffer M, Rubovitch 575 V, Pick CG, Schultz JA, Balaban C (2013) Gangliosides and ceramides change in a mouse model of blast 576 induced traumatic brain injury. ACS Chem Neurosci 4:594-600.

577 Woods AS, Jackson SN (2006) Brain tissue lipidomics: direct probing using matrix-assisted laser 578 desorption/ionization mass spectrometry. AAPS J 8:E391-E395.

579 Woods AS, Kaminski R, Oz M, Wang Y, Hauser K, Goody R, Wang HY, Jackson SN, Zeitz P, Zeitz KP, 580 Zolkowska D, Schepers R, Nold M, Danielson J, Graslund A, Vukojevic V, Bakalkin G, Basbaum A, 581 Shippenberg T (2006) Decoy peptides that bind dynorphin noncovalently prevent NMDA receptor582 mediated neurotoxicity. J Proteome Res 5:1017-1023.

583 Yu Z, Nikolova-Karakashian M, Zhou D, Cheng G, Schuchman E, Mattson M (2000) Pivotal role for 584 acidic sphingomyelinase in cerebral ischemia-induced ceramide and cytokine production, and neuronal 585 apoptosis. J Mol Neurosci 15:85-97. 
Graphical Abstract:
